\title{
Quenching of capillary waves in composite wetting films from a binary vapor: An x-ray reflectivity study
}

\author{
Ralf K. Heilmann, * Masafumi Fukuto, and Peter S. Pershan \\ Division of Engineering and Applied Sciences and Department of Physics, Harvard University, Cambridge, Massachusetts 02138
}

(Received 8 June 2000; published 18 April 2001)

\begin{abstract}
We report x-ray reflectivity measurements of the internal structure of thin wetting films formed on a silicon substrate from the vapor of a binary mixture of methylcyclohexane (MC) and perfluoromethylcyclohexane (PF). At $T=30^{\circ} \mathrm{C}$ (below the bulk consolute temperature of $T_{c} \approx 46^{\circ} \mathrm{C}$ ) variation in the difference in temperature between the substrate and the vapor induces changes in film thickness (25 to $135 \AA$ ) that are consistent with complete wetting of both surfaces, with a MC-rich phase wetting the substrate and a PF-rich phase wetting the free surface. The width of the internal liquid/liquid interface, however, is found to be noticeably smaller than values predicted from the convolution of the intrinsic interfacial profile between bulk liquids with the capillary wave roughness as modified by dispersion interactions with the substrate. The difference is attributed to the effects of confinement. Although the width of the liquid/liquid interface is less well defined above the consolute point $\left(T=60^{\circ} \mathrm{C}\right)$, we still find $\mathrm{PF}$ enrichment at the free surface and MC enrichment at the substrate.
\end{abstract}

DOI: 10.1103/PhysRevB.63.205405

PACS number(s): 68.03. $-\mathrm{g}$, 68.08.Bc

\section{INTRODUCTION}

The physical properties of thin fluid wetting films are important for a variety of processes, such as lubrication, adhesion, catalysis, painting, and coating. In addition, the experimental and theoretical study of wetting phenomena over the last three decades has contributed significantly to the current understanding of statistical physics. ${ }^{1,2}$ When wetting phenomena occur within confined geometries as, for example, in the case of fluids condensing in porous media ${ }^{3-5}$ the interesting new physics involves understanding the competition between the intrinsic bulk properties and the finite length scale of the confinement. The problem becomes more interesting when the unconfined liquid displays critical phenomena, such as for a binary liquid phase in the vicinity of a consolute point. ${ }^{6-8}$

In the present paper we report x-ray reflectivity measurements of wetting films formed on flat $\mathrm{Si}$ substrates from the vapor of a binary mixture of methylcyclohexane (MC) and perfluoromethylcyclohexane (PF). Confinement is between the Si substrate and the free surface of the finite thickness wetting film. The fact that confinement is in one rather than three dimensions as in porous matter simplifies certain aspects of the theoretical problem while allowing direct measurement of fundamental phenomena such as the width of the internal interface between separate phases. This is a difficult quantity to measure and virtually all of the published experimental information concerns the interface between separate phases in binary mixtures of long-chain polymers. ${ }^{9-11}$ In general the observed interfacial widths are smaller than expected and this has motivated a number of theoretical ${ }^{12}$ and computer simulation studies; ${ }^{13-15}$ however, the discrepancies are still unexplained. ${ }^{11}$

The wetting films in the present study are formed from a binary mixture of the comparatively small molecules methylcyclohexane and perfluoromethylcyclohexane. The thickness of these films is controlled through differential heating of the substrate with respect to the surrounding vapor, which in turn is in equilibrium with a liquid reservoir of the mixture. X-ray reflectivity is sensitive to electron densities on angstrom length scales and has been applied successfully to obtain ångstrom level measurements of interfacial structures in numerous liquids and solids. In our system the bulk electron densities of MC and PF differ by close to a factor of 2 , which makes $\mathrm{x}$ rays very well suited to investigate small density or concentration variations.

The much studied binary mixture MC/PF has a conveniently located upper critical solution temperature of $T_{c}$ $\approx 46{ }^{\circ} \mathrm{C}$. ${ }^{16-20}$ At $T \approx 30^{\circ} \mathrm{C}$, in the region of two-phase coexistence, we find clear phase separation within thin wetting films over a range of film thicknesses, with the liquid/liquid interface approximately in the middle of the film. This allows investigation of the interfacial width as a function of distance from the substrate. Similar to the experiments on polymer films we find the width to increase monotonically with the total thickness of the film and thus with the distance between the two confining surfaces. We calculate the expected width as a convolution between the intrinsic width and capillary wave $(\mathrm{CW})$ roughness, assuming that the latter is limited only by long-range van der Waals forces. The width of the intrinsic profile is estimated from an extension of a regular solution model developed by Wynblatt et al. ${ }^{21}$ However, we observe the experimentally determined width to remain smaller than calculated values by factors of 2 to 5 .

The behavior of film thickness versus temperature offset $\Delta T$ between substrate and vapor is consistent with complete wetting of the substrate by an MC-rich phase and of the free surface by a PF-rich phase. This is a direct experimental observation of the thermodynamically stable composite wetting film that was predicted by Evans and co-workers. ${ }^{22,23}$

Above the consolute point, in the single-phase region of the bulk phase diagram at $T \approx 60^{\circ} \mathrm{C}$, our results are less definitive. However, the density profile across the wetting films is still nonmonotonic, indicating that the preferential 
adsorption that exists below the consolute point also prevails far above $T_{c}$.

The rest of this paper is organized as follows: We first describe the experimental setup and measurement technique in Sec. II. Next we discuss some issues relevant for reflectivity modeling and theoretical predictions for the liquid/ liquid and liquid/vapor interfacial widths and wetting behavior for $\mathrm{PF} / \mathrm{MC}$ on $\mathrm{Si}$. We then present the results of measurements of the density profiles, interfacial widths, and wetting behavior, and compare them in Sec. IV with predictions from a regular solution model. We discuss our findings further in Sec. V and summarize in Sec. VI. In the Appendix we present approximation methods to estimate the effects of confinement on $\mathrm{CW}$ roughness.

\section{EXPERIMENTAL SETUP}

\section{A. Wetting chamber and sample preparation}

The experimental design is similar to the one used previously by Tidswell et $a .^{24}$ to study the wetting properties of several liquids. An isothermal chamber is loaded with enough liquid to produce saturated vapors of the substances to be studied. The substrate to be wetted is heated to a higher temperature such that the chemical potential of the liquid in the condensed film is above that of the reservoir. Although the system is not homogeneous across the volume of the cell, the adsorbed film is in thermal equilibrium at well-defined temperature, pressure, and chemical potential, and gradients across the thickness of a film are negligible.

In order to optimize temperature stability and homogeneity, the sample chamber was constructed from two concentric independently temperature controlled cells, surrounded by a further layer of conventional foam insulation. The inner cell, made of oxygen-free high-conductivity copper), contains the substrate and the liquid mixture. The temperature of the ambient vapor, $T$, at the bottom of the inner cell and the temperature of the substrate, $T+\Delta T$, were measured with thermistors that were periodically calibrated with respect to each other. Temperature stability was of the order $\pm 1 \mathrm{mK}$ over a few hours. The uncertainty in $\Delta T$ is conservatively estimated to be no more than $5 \mathrm{mK}$. Two small beryllium windows allow $\mathrm{x}$ rays to enter and exit the inner cell with minimal attenuation. All metallic surfaces are gold coated. Before filling the cell all parts and walls are cleaned with acetone and methanol, followed by a final rinse with methylcyclohexane (95\%). The cell is then slightly heated and blown dry with high-purity Ar gas, and subsequently stored in a glove box under an Ar atmosphere.

The substrate was the (111) surface of a thin Si crystal $\left(32 \times 19 \times 2.5 \mathrm{~mm}^{3}\right)$. Before mounting the substrate undergoes a cleaning cycle consisting of three ultrasonic baths in the order trichloroethylene, acetone, and methanol, followed by rinsing in water, boiling in a sulfuric acid/hydrogen peroxide $\left(70 / 30 \%\right.$ by volume) mixture for 30 minutes at $70^{\circ} \mathrm{C}$, and extensive rinsing in water. ${ }^{25}$ The wafer is then stored under water for short times until the sample cell is ready for mounting. All water used is ultrapure (resistivity $=18.2 \mathrm{M} \Omega \mathrm{cm}$ ). After removing the substrate from the water it is blown dry under a high-purity Ar stream and intro- duced into the glove box, where it is attached to its holder, which is suspended from the lid of the inner cell. Methylcyclohexane (MC, 99\%) and perfluoromethylcyclohexane (PF, 95\%) were purchased from Aldrich Chemical Co. and used without further purification. Before sealing the inner cell with an indium ring it was filled with $3 \mathrm{ml}$ of $\mathrm{MC}$ and 3.125 $\mathrm{ml}$ of $\mathrm{PF}$ at room temperature. With a free inner cell volume of about $115 \mathrm{~cm}^{3}$ we estimate the bulk liquid to be within $1 \%$ of its critical concentration of $x_{c}=50$ vol \% after equilibration at $30^{\circ} \mathrm{C}$. $\mathrm{X}$ rays strike the substrate $4 \mathrm{~cm}$ above the liquid surface.

In a typical experiment the temperature for the inner cell is set and a constant power is applied to the substrate heater. Liquid and vapor equilibrate, saturating the vapor phase, and a wetting film condenses from the vapor onto the substrate. Its thickness is always finite due to the offset in gravitational potential between film and liquid reservoir. However, since the substrate is kept at a temperature $\Delta T$ above $T$, the adsorbed film is always thinner than the value expected from a saturated vapor. The chemical potential difference between wetting film and vapor is determined by $\Delta T$, which in turn fixes the thickness of the wetting film. The temperature on the inside of the cell increases slightly with increasing $\Delta T$. We expect the effects of these shifts in $T$ to have a negligible influence on the thermodynamics of the system, except in the proximity of the critical point.

Measurements begin only after $T$ and $\Delta T$ have not changed over a period on the order of an hour. At that point an x-ray angle of incidence is selected for which the reflectivity is very sensitive to changes in film thickness. It is only after the reflected intensity at that angle has remained constant on the order of an hour that we begin systematic reflectivity measurements. At each $\Delta T$ we perform at least two sets of scans to verify the absence of drifts in either film thickness or composition, which can arise from changes in temperature or from radiation damage to the substrate. With this protocol 2 to 4 values of $\Delta T$ can be measured in a 24 hour period.

\section{B. X-ray measurements}

Specular x-ray reflectivity is sensitive to the average electron density profile along the direction normal to the surface. In a reflectivity measurement the reflected intensity $I$ is measured as a function of the incident angle $\alpha$ to the surface, while keeping the detector at an angle $\beta=\alpha$ to maintain the specular condition (see Fig. 1). The wave vector transfer in vacuum is given as $q=q_{z}=(4 \pi / \lambda) \sin (\alpha)$, with $\lambda$ being the $\mathrm{x}$-ray wavelength.

We collected $\mathrm{x}$-ray reflectivity data from two fillings of the wetting cell at the rotating anode x-ray source of the Harvard Materials Research Laboratory, and from three separate fillings at beamline X22A at the National Synchrotron Light Source (NSLS) at Brookhaven National Laboratory.

Measurements taken at the rotating anode utilized $\mathrm{Cu}$ $K \alpha_{1}$ radiation $(\lambda=1.5405 \AA)$ selected with a $\mathrm{Ge}(111)$ monochromator. The scattering plane and surface normal were horizontal. The incident beam typically was defined by 


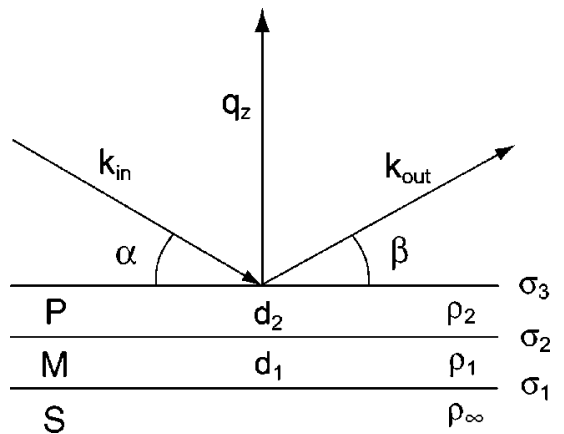

FIG. 1. Scattering geometry for specular reflectivity $(\alpha=\beta)$. The schematically shown stratified model for the density profile along the film normal consists of the semi-infinite substrate $S$ (density $\rho_{\infty}$, roughness $\sigma_{1}$ ), an MC-rich layer $M$ (thickness $d_{1}$, density $\rho_{1}$ ), and a PF-rich layer $P$ (thickness $d_{2}$, density $\rho_{2}$ ). The width of the liquid/liquid interface is given by $\sigma_{2}$, and the width of the liquid/vapor interface by $\sigma_{3}$.

slits of height $2 \mathrm{~mm}$ and width $0.05 \mathrm{~mm}$ at a distance of about $30 \mathrm{~cm}$ before the sample. Detector slits of height $6 \mathrm{~mm}$ and width $1 \mathrm{~mm}$ were situated $30 \mathrm{~cm}$ after the sample.

The wavelength used at beam line X22A of the NSLS was $1.197 \AA$. The beam was focused vertically by a bent mirror. A sagittally focusing $\mathrm{Si}(111)$ monochromator was defocused horizontally for most of the measurements to minimize radiation-induced changes in the wetting properties of the substrate. At X22A the direction of the incident beam is fixed and scattering is measured in the vertical direction. Consequently, in order to vary the angle of incidence relative to the substrate the orientation of the surface normal was varied from pointing upwards $(\alpha=0)$ to no more than $4^{\circ}$ from that direction $\left(q_{z}<0.75 \AA^{-1}\right)$. The incident beam size was defined by slits placed about $407 \mathrm{~mm}$ before the sample (0.05 mm high and $0.6 \mathrm{~mm}$ wide). On the detector side one set of slits $(2 \times 2 \mathrm{~mm})$ at the entrance of an evacuated beam path $181 \mathrm{~mm}$ from the sample served to reduce background scattering. Detector slits of height $1.2 \mathrm{~mm}$ and width $1.0 \mathrm{~mm}$ were placed $530 \mathrm{~mm}$ after the sample. Since the angular spread in the substrate surface normal is negligible compared to the vertical divergence of the incident beam, the latter determines the resolution in $q_{z}$, which we estimate to be $2.6 \times 10^{-4} \AA^{-1}$. In order to remove effects of diffuse scattering from anything other than the sample surface offspecular scattering was measured at $\alpha=\beta \pm 0.1 \mathrm{deg}$ and subtracted from the intensity at the specular condition.

\section{THEORETICAL BACKGROUND}

\section{A. X-ray reflectivity}

When dealing with scattering from liquid surfaces it is important to remember that as a result of thermal excitation the height-height correlations induced by long capillary waves between two points on the surface exhibit a logarithmic dependence on distance that is only terminated through the effects of either gravity ${ }^{26-28}$ or van der Waals attraction between liquid and substrate. ${ }^{29,30}$ This effect has been studied by a number of workers. (References 31 and 32 are some additional examples.) For most reflectivity measurements the experimental conditions are such that the surface structure can be understood as a convolution of the thermal roughness with a suitably defined local structure that is not very different from the way one would interpret the reflectivity in the absence of logarithmic behavior. For more complex layered surfaces the effects can be subtle; however, it is fortunate that these subtle effects are not really important for the measurements to be reported here. A detailed discussion of these effects will be presented elsewhere ${ }^{33}$ however, in order to give the reader some confidence in the present results the basic ideas are sketched below.

In general the specularly reflected intensity $R\left(q_{z}\right)$ from any surface can be calculated from the convolution between a general expression for the differential scattering cross section $d \sigma / d \Omega$ and an instrumental resolution function centered at the specular condition, $q_{x y}=0 .{ }^{26,27}$ If the surface consists of $N$ distinct layers on a substrate (i.e., $N+1$ interfaces), $R\left(q_{z}\right)$ can be represented in the Born approximation (BA) as

$$
\begin{aligned}
\frac{R\left(q_{z}\right)}{R_{F}\left(q_{z}\right)}= & \sum_{i=1}^{N+1}\left[\Theta_{i}\left(q_{z}\right)\right]^{2} e^{-q_{z}^{2} \sigma_{i i}^{2}\left(q_{z}\right)} \\
& +\sum_{i \neq j}^{N+1} \Theta_{i}\left(q_{z}\right) \Theta_{j}\left(q_{z}\right) e^{-q_{z}^{2} \sigma_{i j}^{2}\left(q_{z}\right)} \cos \left(q_{z} d_{i j}\right),
\end{aligned}
$$

where Eq. (1) is normalized to the Fresnel reflectivity $R_{F}\left(q_{z}\right)$ from an ideal substrate/vacuum interface with a step electron density profile. The expression in Eq. (1) is valid for angles $\alpha \geqslant(4-5) \alpha_{c}$, where $\alpha_{c}$ is the critical angle for total external reflection $\left[q_{c}=(4 \pi / \lambda) \sin \left(\alpha_{c}\right)=0.0317 \AA^{-1}\right.$ for $\left.\mathrm{Si}\right]$.

The functions $\Theta_{i}\left(q_{z}\right)$ representing the theoretical scattering amplitude from the $i$ th interface when thermal fluctuations are neglected contain detailed information about the local electron density profile across the interface. The terms $d_{i j}$ denote the average distance along the surface normal between the $i$ th and $j$ th interfaces. The effects of surface roughness, whether static or thermal, are included in the Debye-Waller-like factors

$$
\begin{aligned}
e^{-q_{z}^{2} \sigma_{i j}^{2}\left(q_{z}\right)=} & \frac{1}{(2 \pi)^{2}} \int_{q_{x y} \in q_{r e s}} d^{2} q_{x y} \int_{A} d^{2} r_{x y} \\
& \times e^{i \vec{q}_{x y} \vec{r}_{x y}} e^{-\frac{1}{2} q_{z}^{2}\left\langle\left[u_{i}\left(r_{x y}\right)-u_{j}(0)\right]^{2}\right\rangle},
\end{aligned}
$$

where $q_{\text {res }}$ is the projection of the experimental resolution volume onto the $q_{x y}$ plane and $u_{i}\left(r_{x y}\right)$ describes the fluctuations in the height of interface $i$.

The subtleties referred to earlier have to do with the differences in the way in which the correlation functions $\left\langle u_{i}\left(r_{x y}\right) u_{j}(0)\right\rangle$ behave for different systems. The simplest case might be a surface with a rigid local structure whose position along the surface normal fluctuates because of thermally excited capillary waves. For such a system the different correlation functions are all proportional to one another and the reflectivity can be expressed as ${ }^{34}$

$$
\frac{R\left(q_{z}\right)}{R_{F}\left(q_{z}\right)}=\left|\int_{-\infty}^{\infty} d z \frac{d}{d z}\left[\frac{\langle\rho(z)\rangle}{\rho_{\infty}}\right] e^{-i q_{z} z}\right|^{2},
$$


where $\rho_{\infty}$ is the bulk electron density of the substrate.

For more realistic models $\left\langle u_{i}\left(r_{x y}\right) u_{j}(0)\right\rangle$ $\neq\left\langle u_{k}\left(r_{x y}\right) u_{l}(0)\right\rangle$ and this simple expression cannot be used. When these inequalities are combined with the fact that the resolution area $q_{\text {res }}$ varies with the incident angle, the $q_{z}$ dependence of the various $\sigma_{i j}\left(q_{z}\right)$ Debye-Waller-like factors in Eq. (2) becomes complicated. Fortunately these complications are not important for the present measurements.

The binary MC/PF wetting films on a $\mathrm{Si}$ substrate studied here can be well described by a two-layer, three-interface model. The substrate/liquid interface $(i=1)$ is assumed to be flat with a finite fixed roughness. The fluctuations at the liquid/liquid $(i=2)$ and liquid/vapor $(i=3)$ interfaces and the cross-correlations between them should be determined primarily by the interfacial tensions $\gamma_{i}$ and van der Waals interactions. Quantitiative estimates based on these forces and the relevant experimental parameters indicate that for the range of $q_{z}$ in our measurements the integrals in Eq. (2) can be evaluated by treating $\left\langle u_{i}\left(r_{x y}\right) u_{j}(0)\right\rangle \ll\left\langle u_{i}(0)^{2}\right\rangle,\left\langle u_{j}(0)^{2}\right\rangle$ for all $i, j .{ }^{35}$ Consequently, all of the reflectivity data presented here can be analyzed based on a one-dimensional (1D) average electron density profile $\langle\rho(z)\rangle$. The simplest conceivable model that fits the data well is given by a twobox model consisting of the $\mathrm{Si}$ substrate (electron density $\left.\rho_{\infty}\right)$ and two layers of densities $\rho_{1}$ and $\rho_{2}$ and thicknesses $d_{1}$ and $d_{2}$ for the wetting film. All interfaces are assumed to have error function profiles, consistent with Gaussian roughnesses. We parametrize the density profile as follows:

$$
\begin{aligned}
\frac{\langle\rho(z)\rangle}{\rho_{\infty}}= & 1+\frac{1}{2}\left(\phi_{1}-1\right)\left[1+\operatorname{erf}\left(\frac{z}{\sqrt{2} \sigma_{1}}\right)\right] \\
& +\frac{1}{2}\left(\phi_{2}-\phi_{1}\right)\left[1+\operatorname{erf}\left(\frac{z-d_{1}}{\sqrt{2} \sigma_{2}}\right)\right] \\
& -\frac{1}{2} \phi_{2}\left[1+\operatorname{erf}\left(\frac{z-\left(d_{1}+d_{2}\right)}{\sqrt{2} \sigma_{3}}\right)\right],
\end{aligned}
$$

where $\phi_{i}=\rho_{i} / \rho_{\infty}, \sigma_{1}$ is the roughness for the substrate/ liquid interface, $\sigma_{2}$ the roughness for the liquid/liquid interface between the two layers, and $\sigma_{3}$ the roughness for the liquid/vapor interface. In this model, both the intrinsic interfacial width and the mean-square fluctuations of the interfacial height of interface $i$ are contained in the roughness parameter $\sigma_{i}$.

At $T=30{ }^{\circ} \mathrm{C}$ the relative bulk densities for the pure liquids $\phi_{P F}$ and $\phi_{M C}$ are 0.730 and 0.374 , respectively. Based on the phase diagram by Heady and Cahn ${ }^{17}$ the corresponding values for the coexisting phases in the bulk mixture are 0.677 (PF-rich) and 0.424 (MC-rich), i.e., there is good contrast to detect phase separation with $\mathrm{x}$-ray measurements. Layer thicknesses can be extracted from the periods of characteristic oscillations (Kiessing fringes) in the reflectivity.

If the wetting film covering the substrate were homogeneous, then for the materials properties just discussed the single value of $\phi_{1}=\phi_{2}=\phi<1$. It is straightforward to demonstrate that for $\phi<1$ the maximum value of the ratio $R\left(q_{z}\right) / R_{F}\left(q_{z}\right)=(1-2 \phi)^{2} \leqslant 1$. On the other hand, if the wet- ting film consists of two distinguishable layers $R / R_{F}>1$ is only possible if $\phi_{2}>\phi_{1}$. The conclusion is that for this model, observation of $R / R_{F}>1$ for any $q_{z}$ implies that the electron density profile is nonmonotonic.

In our films $\rho(z)$ varies over distances comparable to $q_{c}^{-1}$, and the reflectivity contains important information close to the critical angle. For quantitative data analysis we therefore employ the Parrat formalism, ${ }^{36}$ where $q_{z}$ is a complex number that depends on the index of refraction and absorption in each layer. This formalism remains valid close to the critical angle. We use two slightly different methods to implement it. In the first method (method I) the density profile is represented as a two-box model with sharp interfaces. Interfacial roughness is included through multiplication of reflected and transmitted waves by terms $\propto \exp$ $\left(-q_{z, i} q_{z, i+1} \sigma_{i}^{2}\right)$, with $q_{z, i}$ and $q_{z, i+1}$ being the complex wavevector transfers in the layers bordering interface $i{ }^{26,37}$ This treatment becomes inaccurate when the roughnesses of neighboring interfaces are comparable to or greater than the thickness of the layer that separates them. ${ }^{38}$ In such cases we parametrize the density profile according to Eq. (4), approximate the profile by splitting it into $1 \AA$ thick slabs of constant density and zero roughness, and apply the exact Parrat method to obtain the physically accurate reflectivity (method II). For a $40 \AA$ thick film the latter method thus calculates reflection and transmission for more than 40 interfaces, while method I uses only three interfaces.

While for small $q_{z}$ the Parrat formalism is more accurate than the Born approximation, the above discussion of qualitative features in $R / R_{F}$ remains valid in the Parrat formalism.

\section{B. The liquid/liquid interface}

Most of our measurements were performed at $T \approx 30^{\circ} \mathrm{C}$, where the $\mathrm{PF} / \mathrm{MC}$ mixture has a wide miscibility gap. In the following we therefore describe theoretical expectations for the liquid/liquid interface that forms when the binary liquid separates into a MC-rich and a PF-rich phase.

For a bulk binary solution, as a result of the lower surface tension a PF-rich phase wets the MC-rich/vapor interface ${ }^{17}$ against gravity despite its higher mass density. This behavior is typical for hydrocarbon and perfluorocarbon mixtures, and it can be explained by a mean field theoretical approach from the lower molecular number density of the phase rich in the perfluorocarbon. ${ }^{39}$ In addition MC has been found to wet the $\mathrm{PF}-$ rich $/ \mathrm{SiO}_{2} / \mathrm{Si}$ interface ${ }^{40}$ in bulk mixtures. It is therefore reasonable to assume that a wetting film formed from the binary vapor could phase separate into an MC-rich layer at the solid substrate and a PF-rich layer at the liquid/vapor interface, thereby forming a composite wetting film ${ }^{22}$ with an internal liquid/liquid interface.

The average profile of this interface can be represented as a convolution of profiles reflecting the intrinsic width $w_{0}$ and roughness due to thermal capillary waves. The intrinsic density profile across the interface between bulk phases of a binary mixture is well approximated by an error function of width $\approx 2 \xi_{b}$, where $\xi_{b}$ is the bulk correlation length of the mixture. ${ }^{41,42}$ For the mixture PF/MC the bulk correlation length $\xi_{0}^{+}$above $T_{c}$ has been determined from ellipsometry 
measurements to be $2.79 \AA .{ }^{19}$ Using the universal ratio for the 3D Ising model $\xi_{0}^{+} / \xi_{0}^{-}=1.96$ (Ref. 43) and the scaling relation ${ }^{44} \xi_{b}=\xi_{0}^{-}\left(\left|T-T_{c}\right| / T_{c}\right)^{-0.63}$ we obtain $\xi_{b}=9.32 \AA$ at $T=30^{\circ} \mathrm{C}$.

The $\mathrm{CW}$-induced roughness is estimated by integrating the mean-square amplitude of $\mathrm{CW}$ modes with in-plane surface wave vectors $\left\{\mathbf{q}_{x y}\right\}$ :

$$
\sigma_{\mathrm{CW}}^{2}=\frac{k_{B} T}{4 \pi^{2}} \int_{q_{\min }}^{q_{\max }} \frac{d^{2} \mathbf{q}_{x y}}{\gamma\left(\mathbf{q}_{x y}^{2}+\xi_{\|}^{-2}\right)}=\frac{k_{B} T}{4 \pi \gamma} \ln \left(\frac{q_{\max }^{2}+\xi_{\|}^{-2}}{q_{\min }^{2}+\xi_{\|}^{-2}}\right),
$$

where $k_{B}$ is the Boltzmann constant, $\gamma$ is the interfacial stiffness, and $\xi_{\|}$is a characteristic length at which capillary waves become quenched by an external potential. It is due to gravity in bulk liquids and van der Waals interactions with the substrate for thin wetting films. The validity of an equivalent description has been demonstrated in recent $\mathrm{x}$-ray scattering experiments on the liquid/liquid interface in a bulk mixture of hexane and perfluorohexane. ${ }^{42}$ For thin wetting films $\xi_{\|}$is given by ${ }^{1}$

$$
\xi_{\|}=d_{1}^{2}\left(\frac{2 \pi \gamma}{A_{\text {eff }}}\right)^{1 / 2}
$$

where $d_{1}$ is the distance between interface and substrate and $A_{\text {eff }}$ is the effective Hamaker constant for positional fluctuations of the interface around a potential minimum. ${ }^{45} \mathrm{We}$ discuss and quantitatively estimate $A_{\text {eff }}$ further below. The lower integration limit, $q_{\min }$, is defined by the projection of the $\mathrm{x}$-ray coherence area onto the surface. Its value varies with the angle of incidence, but is typically on the order of micrometers. The value of $q_{\max }$ depends on the statistical properties of the surface. For noncritical liquids the maximum is usually taken to be the value for which the total number of surface modes corresponds to the number of surface atoms, i.e., $\propto 2 \pi / B_{0}$, where $B_{0}$ is the size of a liquid molecule. However, for liquids exhibiting critical correlations a better value is probably $2 \pi / \xi_{b}$, where the effects of fluctuations with shorter wave vector are contained in the intrinsic width. For PF/MC $B_{0} \approx 6 \AA$, which is comparable to the value for $\xi_{b}$ at $30^{\circ} \mathrm{C}$ estimated above. We will show below that for the present experiments $2 \pi / q_{\min } \gg \xi_{\|} \gg B_{0}$, except for the thinnest films, where $\xi_{\|} \sim B_{0}$.

Assuming that both $w_{0}$ and the contribution from capillary waves can be represented by error functions, the total width arising from the convolution of both profiles can thus be described by

$$
\sigma^{2}=w_{0}^{2}+\frac{k_{B} T}{2 \pi \gamma} \ln \left[\frac{d_{1}^{2}}{B_{0}}\left(\frac{\gamma}{A_{\text {eff }}}\right)^{1 / 2}(2 \pi)^{3 / 2}\right] .
$$

As a plausible estimate for $\gamma$ we use the average of two measurements of the interfacial tension in the bulk mixture, ${ }^{17,18} 0.58 \mathrm{mN} / \mathrm{m}$. To estimate $A_{\text {eff }}$ we consider a stratified system consisting of the semi-infinite substrate $(S)$, a layer of pure $\mathrm{MC}\left(M\right.$, thickness $\left.d_{1}\right)$ and another layer of pure PF $\left(P\right.$, thickness $\left.d_{2}\right) .{ }^{46}$ Thermal fluctuations in the interfacial position affect $d_{1}$ and $d_{2}$. We are therefore inter-
TABLE I. Simple estimates for Hamaker constants from Lifshitz theory (Ref. 47) and from surface tensions at $30^{\circ} \mathrm{C}$. The static dielectric constants and characteristic UV absorption frequencies used in the Lifshitz theory were taken from Ref. 40. F refers to $\mathrm{SiO}_{2}$.

\begin{tabular}{ccc}
\hline \hline$i j$ & $A_{i j}\left(10^{-20} \mathrm{~J}\right)^{\mathrm{a}}$ & $A_{i j}\left(10^{-20} \mathrm{~J}\right)^{\mathrm{b}}$ \\
\hline$S M$ & 17.4 & - \\
$M M$ & 4.92 & 4.86 \\
$M P$ & 3.85 & - \\
$P P$ & 2.96 & 2.63 \\
$S P$ & 13.9 & - \\
$F P$ & 4.9 & - \\
$F M$ & 6.2 & - \\
\hline \hline
\end{tabular}

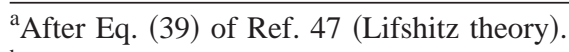

${ }^{\mathrm{b}}$ After Eq. (11.35) of Ref. 45 (surface tension).

ested in the $\left(d_{1}, d_{2}\right)$ dependence of the excess free energy per unit area of this system, which can be written in the form

$$
\begin{aligned}
G\left(d_{1}, d_{2}\right)= & \frac{1}{12 \pi}\left(\frac{A_{S M}-A_{S P}+A_{M P}-A_{M M}}{d_{1}^{2}}+\frac{A_{S P}-A_{M P}}{\left(d_{1}+d_{2}\right)^{2}}\right. \\
& \left.+\frac{A_{M P}-A_{P P}}{d_{2}^{2}}\right) .
\end{aligned}
$$

$A_{i j}$ is the Hamaker constant for the interaction across vacuum between two bodies consisting of components $i$ and $j$, respectively. ${ }^{45}$ When expanding $G$ for small fluctuations $u \equiv d_{1}-d_{1, \text { min }}$ in the position of the M/P interface $d_{1}$ around a potential minimum $d_{1, \min }$ with $d_{1}+d_{2}$ fixed one obtains

$$
\delta G(u)=G(u)-G(0) \approx \frac{1}{2} \frac{\partial^{2} G}{\partial u^{2}} u^{2}=\frac{1}{4 \pi} \frac{A_{\mathrm{eff}}}{d_{1, \min }^{4}} u^{2},
$$

where the effective Hamaker constant that determines the energy per unit area associated with small fluctuations in $d_{1}$ is given by

$$
A_{\mathrm{eff}}=A_{S M}-A_{S P}+A_{M P}-A_{M M}+\left(\frac{d_{1}}{d_{2}}\right)^{4}\left(A_{M P}-A_{P P}\right),
$$

i.e., $A_{\text {eff }}$ depends on the ratio of the two film thicknesses. In Table I we compare estimates for the $A_{i j}$ from surface tensions ${ }^{45}$ and Lifshitz theory. ${ }^{47}$ We show below that these values of $A_{i j}$ imply that $d_{1} \approx d_{2}$. With $d_{1}=d_{2}$ and our choices of $A_{i j}$ 's one obtains $A_{\text {eff }}=3.3 \times 10^{-20} \mathrm{~J}$. This leads to a CW roughness of $10-25 \AA$ and puts $\xi_{\|}$between 3.3 and $330 \AA$ for $d_{1}$ between 10 and $100 \AA$.

The $\mathrm{CW}$ roughness given by the second term in Eq. (7) is based on the leading $u^{2}$ term in the expansion for the van der Waals potential $\delta G(u)$ [Eq. (9)]. As such it is only accurate when the interfacial width is significantly smaller than the distances between the interface and the confining "hard walls." In the Appendix we attempt to take into account the higher-order terms in the expansion of $\delta G(u)$ in order to estimate the effects of confinement on $\mathrm{CW}$ roughness. 


\section{Wetting film thickness and the liquid/vapor interface}

The Hamaker approach can also be used to make predictions about wetting film thickness ${ }^{48}$ and the width of the liquid/vapor interface. For this purpose we add two terms to the excess free energy of Eq. (8). The first term is due to the chemical potential differential arising from the offset between the temperature of the substrate and that of the solution $\Delta \mu_{i}=\left(\partial \mu_{i} / \partial T\right)_{P} \Delta T \approx H_{i} \Delta T / T$, where $i, j=1,2$ for MC and $\mathrm{PF}$, respectively, and $H_{i}$ is the latent heat of vaporization per molecule of component $i{ }^{24}$ The second term arises from the gravitational energy. The total excess free energy per unit area is now given by

$$
\begin{aligned}
G\left(d_{1}, d_{2}, h, \Delta T\right)= & G_{0}\left(d_{1}, d_{2}\right)+d_{1}\left(n_{1} H_{1} \Delta T / T+g h \rho_{1}\right) \\
& +d_{2}\left(n_{2} H_{2} \Delta T / T+g h \rho_{2}\right),
\end{aligned}
$$

with $n_{i}$ being the molecular number densities, $h$ the height of the film above the liquid reservoir, and $\rho_{i}$ the mass densities. The equilibrium thicknesses are obtained from $\partial G / \partial d_{i}=0$ ( $i=1,2)$. Defining $c \equiv d_{2} / d_{1}$ the two resulting equations can be solved for $c$ and one obtains for the total film thickness

$$
\begin{aligned}
l & =d_{1}+d_{2} \\
& =d_{1}(1+c) \\
& =\left(\frac{\left(A_{S M}-A_{S P}+A_{M P}-A_{M M}\right)(1+c)^{3}+A_{S P}-A_{M P}}{6 \pi\left(H_{1} n_{1} \Delta T / T+g h \rho_{1}\right)}\right)^{1 / 3} .
\end{aligned}
$$

With the values from Table I $c$ ranges from $0.945(\Delta T$ $\rightarrow \infty)$ to $0.859(\Delta T=5 \mathrm{mK})$, i.e., the liquid/liquid interface is expected to be located close to the middle of the wetting film. For large values of $\Delta T$ where the gravitational energy can be neglected the film thickness depends on $\Delta T$ as $l$ $\propto \Delta T^{-1 / 3}$, as expected for van der Waals interactions with the substrate.

The liquid/vapor interface is also broadened due to capillary excitations. The surface tensions for the pure bulk liquids are $12.5 \mathrm{mN} / \mathrm{m}$ and $23.15 \mathrm{mN} / \mathrm{m}$ for $\mathrm{PF}$ and $\mathrm{MC}$, respectively, roughly 20-40 times greater than at the liquid/ liquid interface. According to Eq. (5) the CW roughness at the liquid/vapor interface is therefore much smaller than at the confined interface. We consequently neglect positional correlations between the two interfaces. Thus the effective Hamaker constant for fluctuations at the free surface can be written as

$$
A_{\mathrm{eff}} \approx 4\left(A_{S M}-A_{M M}+A_{M P}-A_{P P}\right)+3\left(A_{M P}-A_{S P}\right),
$$

where we used $c \approx 1$ for simplicity. The value of $\sigma_{3}$ would change by less than $1 \%$ if we were to use the exact values for c.

The intrinsic width of the liquid/vapor interface for dielectric bulk liquids ${ }^{49}$ and thin wetting films ${ }^{32}$ is typically found to be zero or negligible compared to $\mathrm{CW}$ roughness. Studies on high-surface-tension liquid metal surfaces, where $\mathrm{CW}$ roughness is even more reduced, found intrinsic widths of only $0.3-0.37 \AA .{ }^{50}$

\section{RESULTS}

For the range of $q_{z}$ over which our data were taken we are not compelled to include an expected $10-15 \AA$ thin native oxide layer in the model for our substrate for two reasons. First is the empirical fact that data from both the bare and the wetted substrate can be modeled with or without a suitably rough oxide layer, with any differences for the wetting film parameters well inside the error bars shown. Second is the practical fact that data from our samples are not necessarily expected to be sensitive to the presence of a thin oxide layer. The electron density of $\mathrm{SiO}_{2}$ is within $3 \%$ of that of pure $\mathrm{Si}$, which leads to relatively weak interference fringes. Also the fringe period would be very large due to the expected thinness of the oxide. In addition the measured reflectivity data from the bare substrate fall off with increasing angle, indicative of an rms surface roughness on the order of $3.5 \AA$. This means that both the overall reflectivity and the amplitude of interference effects due to a thin oxide layer become small at such small angles of incidence that the data presented here are rather insensitive to the presence or absence of native oxide.

\section{A. Density profiles at $\boldsymbol{T}=30^{\circ} \mathrm{C}$}

Figure 2(a) shows a set of reflectivities from a single filling, measured sequentially at $T \simeq 30^{\circ} \mathrm{C}$ with increasing $\Delta T$. The rapid oscillations (Kiessing fringes) are due to interference between reflections from the solid/liquid and liquid/ vapor interfaces. As $\Delta T$ is increased the period of these fringes increases, i.e. the film on the substrate becomes thinner. In all but the thinnest films $\left(d_{1}+d_{2} \geqslant 30 \AA\right)$ the normalized reflectivity $R / R_{F}$ exceeds 1 at the first maximum for $q_{z}>q_{c}$. As discussed in Sec. III A, the $\phi_{i}$ for both liquid components are smaller than one, and the wetting film therefore must have a nonmonotonic density profile. Thus the simplest model to allow $R / R_{F}>1$ consists of 2 layers, with $\phi_{1}<\phi_{2}$, in agreement with MC being favored at the substrate and PF at the free surface of the wetting film.

Table II gives the best fitting parameters for the two-box model. The confidence limits are taken from the size of the nonlinear support plane in the space of fitting parameters. For our model we find these error estimates to be more realistic than the conventional error bars derived from the variation of a single parameter, ${ }^{51}$ which are 3-5 times smaller in our case.

In Fig. 2(b) we show the density profiles from the best fits of the two-box model to our data. All three profiles show a composite wetting film with a PF-rich layer at the free surface and an MC-rich layer at the substrate, in qualitative agreement with expectations. The density of the MC-rich layer coincides with the value for pure MC. The difference $\phi_{2}-\phi_{1}$ is close to $0.20 \pm 0.02$. This is smaller than 0.253 , the corresponding value from the bulk phase diagram. For the other fillings best fits for films of similar thickness give $\phi_{1}$ in the range $0.36-0.44$ and $\phi_{2}-\phi_{1}$ between 0.21 and 0.24 . The PF-rich layer is thus found to be less dense than in a bulk mixture.

For all but the thinnest films the best fit values for the width, $\sigma_{2}$, of the liquid/liquid interface is rather large at 10- 


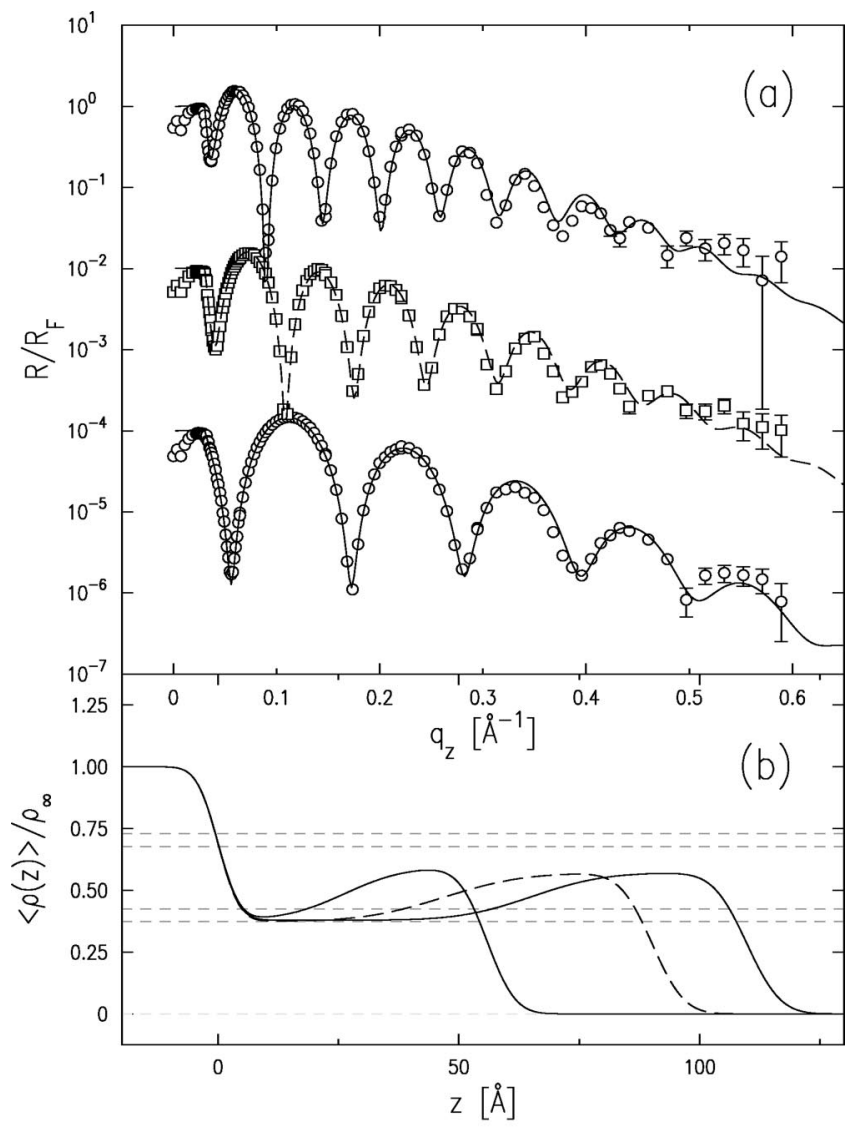

FIG. 2. (a) Normalized reflectivities at $\Delta T \approx 7.5,39$, and 214 $\mathrm{mK}$ (top to bottom, $T \approx 30^{\circ} \mathrm{C}$ ). Lines are two-box model fits using method I as described in the text. (b) Density profiles from fits to the data in (a). The gray dashed lines mark the relative bulk densities for pure PF, the PF-rich, and the MC-rich phase at $30^{\circ} \mathrm{C}$, and pure MC (top to bottom).

$15 \AA$. The results for $\sigma_{2}$ are shown in Fig. 3. As a consequence of the large interfacial width, the effect of the interface on the reflectivity is confined to small values of $q_{z}$, and the precision with which the difference $d_{1}-d_{2}$ can be determined is considerably less than that of the sum $d_{1}+d_{2}$. Nevertheless, it is clear that for all of the data the position of the interface is close to the center of the film.

The plots in Fig. 4 illustrate the manner in which information on the liquid/liquid interface is manifest in the data. The open circles are a typical data set taken at $T=30^{\circ} \mathrm{C}$. We

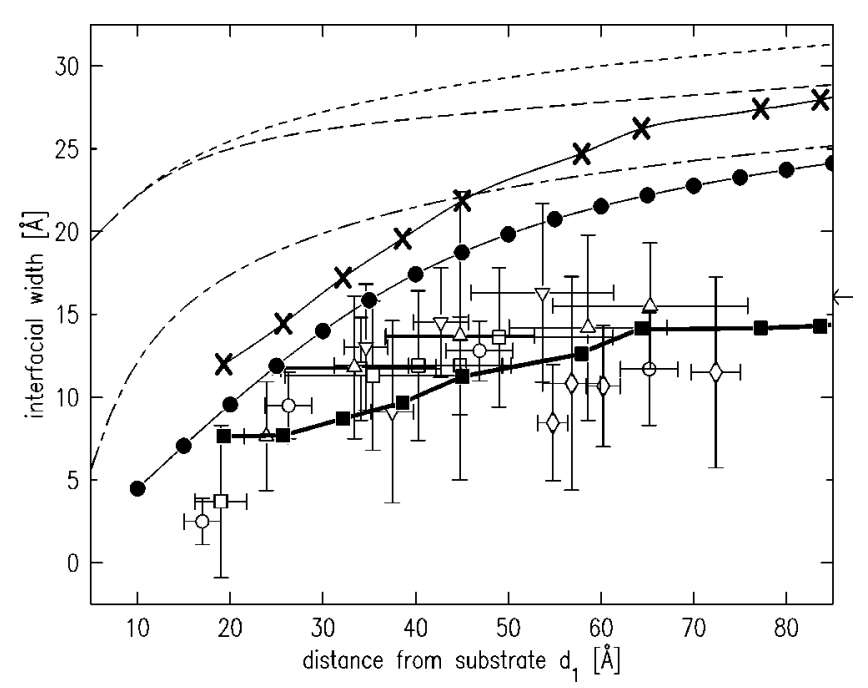

FIG. 3. Width of the liquid/liquid interface $\left(\sigma_{2}\right)$ as a function of distance from the substrate at $T \approx 30{ }^{\circ} \mathrm{C}$ (method I). Open circles, squares, and diamonds are from synchrotron data, and open triangles from rotating anode data. The two topmost dashed lines show predictions according to Eq. (7) with $\gamma=0.58 \mathrm{mN} / \mathrm{m}, B_{0}$ $=6.0 \AA$, and $w_{0}=2 \xi_{b}=18.6 \AA$. The top line considers long-range interactions only, while the line below it includes both long- and short-range interactions (Ref. 15). The third line from the top shows the contribution from $\mathrm{CW}$ roughness only $\left(w_{0}=0\right)$, but otherwise uses the same values as the topmost line. All three lines use $A_{\text {eff }}$ $=3.3 \times 10^{-20} \mathrm{~J}$. The filled squares are results for the intrinsic width $w_{0}\left(d_{1}\right)$ based on the regular solution model, and the arrow at the right axis marks $w_{0}(\infty)=16.15 \AA$ (see text). The filled circles show the self-consistent $\mathrm{CW}$ roughness alone based on Eqs. (A14) and (A19) and include the effects of higher-order terms of a long-range van der Waals potential (see the Appendix). The crosses, which are the convolution of the data shown by filled squares and filled circles, represent the theoretically expected interfacial width.

show the best fit with all parameters varied and the best fit with $\sigma_{2}$ held at specific values.

For values of $\sigma_{2}$ between 7 and $25 \AA$ the reflectivity is sensitive to the presence of the liquid/liquid interface only in the region of $q_{z}$ below about 0.1 to $0.15 \AA^{-1}$. Increasing $\sigma_{2}$ beyond the confidence limit given by the error bar in Fig. 3 leads to a visible deterioration in the match between data and fit.

For the thinnest films $\left(d_{1}+d_{2} \leqslant 40 \AA\right)$ we find different sets of fitting parameters that lead to fits of comparable qual-

TABLE II. Fit parameters for the fits shown in Figs. 2 and 5 (method I). $\sigma_{1}$ was held fixed at $3.5 \AA$. For the thinnest film two sets of parameters are given. The first set is from fitting to data with $q_{z} \geqslant 0.15 \AA^{-1}$ only and is depicted in Fig. 5. The second set is from a fit to $q_{z} \geqslant 0.07 \AA^{-1}$, which fits the first minimum very well, but lies too low at $q_{z}>0.4 \AA^{-1}$ (not shown).

\begin{tabular}{rrccccc}
\hline \hline$d_{1}+d_{2}(\AA)$ & $d_{1}-d_{2}(\AA)$ & $\phi_{1}$ & $\phi_{2}-\phi_{1}$ & $\sigma_{1}(\AA)$ & $\sigma_{2}(\AA)$ & $\sigma_{3}(\AA)$ \\
\hline $109.8 \pm 0.2$ & $21 \pm 6$ & $0.379 \pm 0.017$ & $0.191 \pm 0.029$ & 3.5 & $11.7 \pm 3.3$ & $5.5 \pm 0.5$ \\
$90.1 \pm 0.2$ & $4 \pm 7$ & $0.375 \pm 0.012$ & $0.194 \pm 0.023$ & 3.5 & $12.8 \pm 1.8$ & $5.2 \pm 0.4$ \\
$55.8 \pm 0.3$ & $-3 \pm 5$ & $0.382 \pm 0.015$ & $0.209 \pm 0.027$ & 3.5 & $9.5 \pm 2.0$ & $4.6 \pm 0.4$ \\
$34.0 \pm 0.2$ & $-5 \pm 5$ & $0.45 \pm 0.10$ & $0.14 \pm 0.10$ & 3.5 & $1.3 \pm 2.0$ & $3.7 \pm 0.4$ \\
$33.9 \pm 0.2$ & $0 \pm 4$ & $0.504 \pm 0.012$ & $0.136 \pm 0.020$ & 3.5 & $2.5 \pm 1.4$ & $3.4 \pm 0.3$ \\
\hline \hline
\end{tabular}




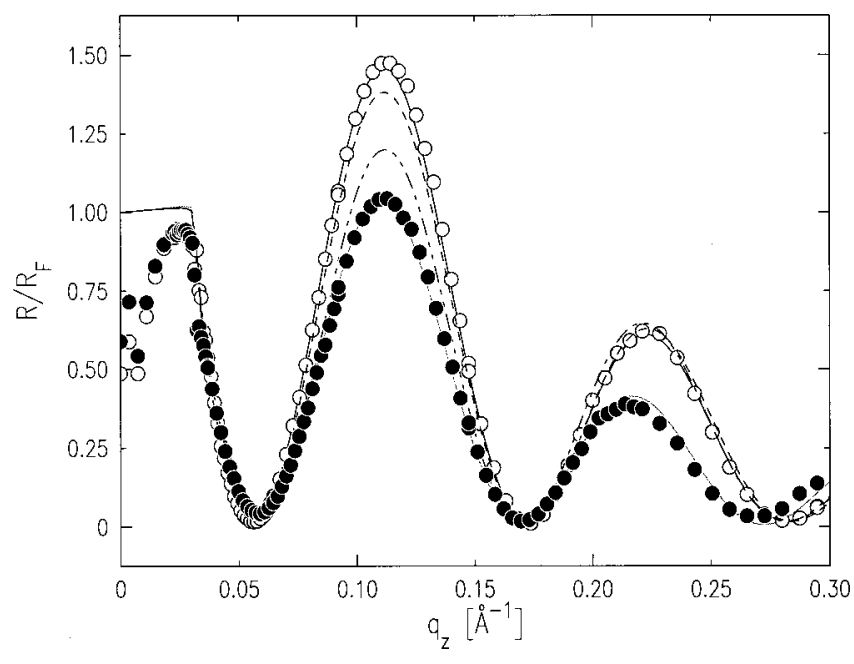

FIG. 4. $R / R_{F}$ from a film at $T \approx 30^{\circ} \mathrm{C}$ (open circles) and best fit [solid line, $\sigma_{2}=9.6 \AA,\left(\phi_{2}-\phi_{1}\right) \approx 0.21$ ], and from a film at $T$ $\approx 60{ }^{\circ} \mathrm{C}$ (closed circles) and best fit (gray line). The dashed line is a best fit to the $30^{\circ} \mathrm{C}$ data with $\sigma_{2}$ held fixed at $12 \AA$, and the dash-dotted line uses $\sigma_{2}=25 \AA$. Using $\sigma_{2}>25 \AA$ gives essentially the same fit as the dash-dotted line, since for this width reflections from the liquid/liquid interface are negligible beyond $q_{z}$ $\approx 0.08 \AA^{-1}$. In order to fit the $60{ }^{\circ} \mathrm{C}$ data, $\phi_{2}-\phi_{1}$ has to be reduced below 0.1. In all cases the data are not fitted for $q_{z}$ $<0.035 \AA^{-1}$, since for those values the sample does not fully intercept the incident beam.

ity. Figure 5(a) shows $R / R_{F}$ for a $34 \AA$ thick film. The solid line is an example of a fit using method I resulting in $\sigma_{2}$ $=1.3 \AA$. The fit at the first minimum in $R / R_{F}$ can be improved by increasing $\sigma_{2}$ to values where the different interfaces are not separable anymore and method I becomes unphysical. At the same time the fit then significantly underestimates $R$ at larger $q_{z}$. The dashed line is a fit using method II. It matches the data very well up to $q_{z}$ $\approx 0.4 \AA^{-1}$, but lies below the data for larger $q_{z}$. The density profiles for both fits are shown in Fig. 5(b). While the physical results for method I show clear phase separation, method II leads to an almost linear increase in density from a minimum close to the substrate to a maximum close to the free surface without any region of constant density. The true density profile probably lies somewhere between the two models shown and cannot be parametrized according to Eq. (4). The density difference between local minimum and maximum is smaller than in the thicker films, and it is found to decrease with decreasing film thickness.

To summarize, the results show that the substrate is wetted by a composite film with an approximately pure MC layer at the substrate and a PF-rich layer at the free surface. The best fits place the interface between the two liquids halfway between the free surface and the substrate.

\section{B. The liquid/liquid interface}

Best fit results from method I for the width of the liquid/ liquid interface, $\sigma_{2}$, are shown in Fig. 3 as a function of distance from the substrate, $d_{1}$. Despite the large uncertainties an increase in $\sigma_{2}$ with increasing $d_{1}$ is discernible.

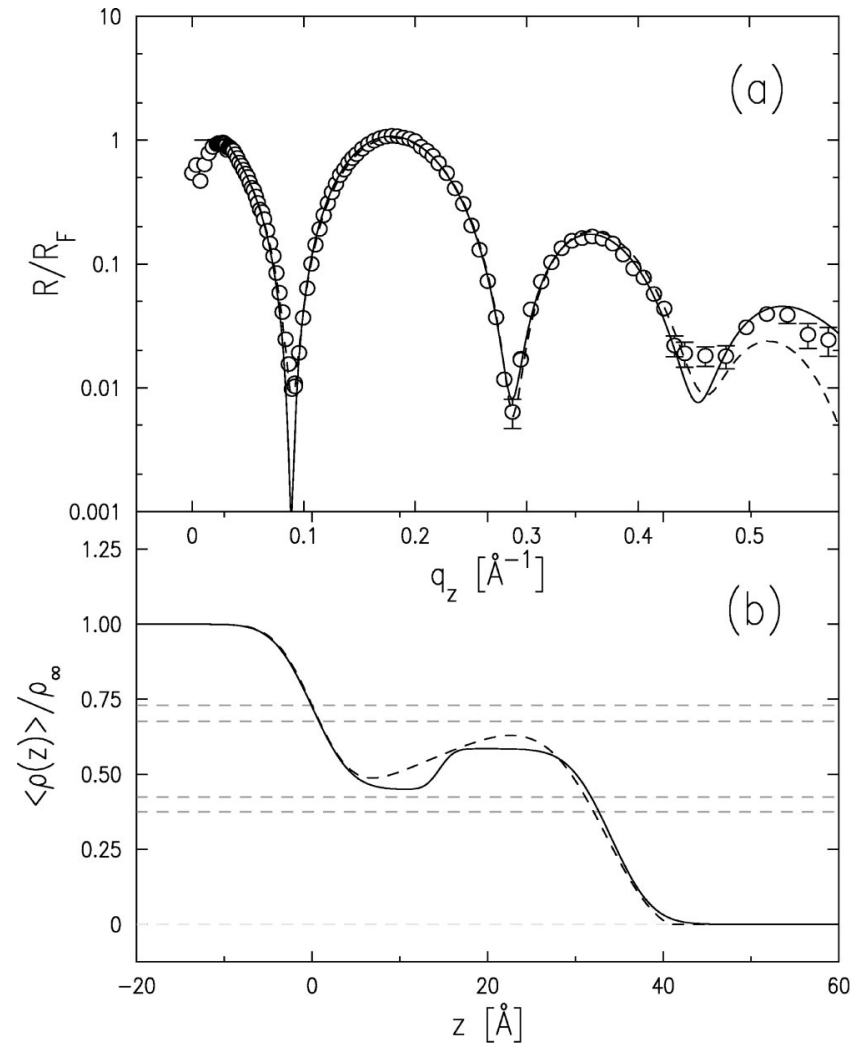

FIG. 5. (a) Normalized reflectivities at $\Delta T \approx 963 \mathrm{mK}(T$ $\approx 30{ }^{\circ} \mathrm{C}$ ). The solid line is a two-box model fit using method I, while the dashed line shows a fit using method II as described in the text. (b) Density profiles from fits to the data in (a). The solid line is from method I, and the dashed line from method II. Again the gray dashed lines mark the relative bulk densities for pure PF, the PFrich and the $\mathrm{MC}$-rich phases at $30^{\circ} \mathrm{C}$, and pure $\mathrm{MC}$ (top to bottom).

We also show predictions from Eq. (7) with $w_{0}$ $=18.6 \AA$ and for comparison with $w_{0}=0$. The discrepancy between theoretical predictions and experimental results is pronounced. In order to reproduce the experimental data both intrinsic width and capillary wave roughness would have to be smaller than predicted, since both contributions by themselves as calculated above exceed the measured values. Also illustrated in Fig. 3 is the effect of adding short-range potentials as discussed by Binder and co-workers. ${ }^{11,15}$ The discrepancy is not significantly reduced. Entropic terms that limit the fluctuations at the confined interface as described by Ross et al. ${ }^{52}$ are negligible in our system for $d_{1} \geqslant 10 \AA$. The filled circles in Fig. 3 show the $\mathrm{CW}$ part of the width when higher-order terms in the van der Waals potential $\delta G(u)$ are taken into account (see Appendix); it predicts reduced $\mathrm{CW}$ roughness, but the results still lie above the measured values even for $w_{0}=0$. One possible reason for the differences between theoretical predictions and our measurements could be that either $\gamma$ or $w_{0}$ somehow depend on film thickness and are therefore not equal to their values for $d_{1}, d_{2} \rightarrow \infty$. As argued previously, ${ }^{11,12}$ it is intuitively clear that $w_{0}$ has to be limited by the film thickness, and it is reasonable to assume that $w_{0}(l)$ already differs from the bulk value $w_{0}(\infty)$ for some value of $l=d_{1}+d_{2}>w_{0}(\infty)$. An increase in the inter- 
facial stiffness $\gamma$ with decreasing film thickness could also contribute to the observed behavior. Recent computer simulations of the confined interface of a binary polymer mixture were interpreted in terms of just this effect. ${ }^{13,14}$ Although for our system this does not appear to be a likely explanation for films over $200 \AA$ thick (i.e., $6-7$ times the predicted interfacial width) the effect has to be considered.

In an attempt to better estimate how confinement affects capillary waves and $w_{0}$ separately, we calculated the intrinsic width by using the regular solution model for a binary mixture developed by Wynblatt et al. ${ }^{21}$ for binary metallic alloys. The model assumes a finite internal energy from nearest-neighbor interactions with bond energies $u_{i j}(i, j$ $=M, P)$ and an ideal solution entropy. It consists of a finite number $(n)$ of layers of fixed molecular number density, where each molecule has six nearest neighbors within the layer and three nearest neighbors in each of the two neighboring layers. Each layer is characterized by its composition $x_{k}(k=1, \ldots, n)$, which we take as the molecule fraction of $\mathrm{PF}$, or $P$ in short. The surface excess free energy is calculated layer by layer and minimized through variation of the $x_{i}$. We extend this model through the addition of a van der Waals energy $\propto u_{i S} / k^{3}$ due to the interaction between substrate (next to layer 1) and the $k$ th layer. The chemical potential difference between the reservoir and the adsorbed film due to $\Delta T \neq 0$ is taken into account through $L_{i} \Delta T / T,{ }^{24}$ where $L_{i}$ is the molar latent heat of vaporization for component $i$. Each layer contributes a potential energy term $\left[\rho_{P} x_{k}\right.$ $\left.+\rho_{M}\left(1-x_{k}\right)\right] g \Delta h$, with $\rho_{i}$ being molar mass densities, $g$ $=9.81 \mathrm{~m} / \mathrm{s}^{2}$, and $\Delta h$ is the height of the film above the liquid reservoir. We estimate $u_{M M}=-3833 \mathrm{~J} / \mathrm{mol}$ and $u_{P P}$ $=-2083 \mathrm{~J} / \mathrm{mol}$ from the surface tensions $\gamma_{i}$ and the average of the areal molecular densities for the pure liquids $\Omega$ via $u_{i i}=-2 \Omega \gamma_{i} / 3 .^{21}$ Setting $T_{c}=319 \mathrm{~K}$ and using $T_{c}=\left(6 / R_{g}\right)$ $\times\left[u_{M P}-\left(u_{M M}+u_{P P}\right) / 2\right]$, with $R_{g}$ being the gas constant, then determine that $u_{M P}=-2516 \mathrm{~J} / \mathrm{mol}$, i.e., the MC-PF interaction is about $10 \%$ weaker than the geometric mean of the interaction between like molecules. ${ }^{16}$ We further use $u_{M S}=-5559 \mathrm{~J} / \mathrm{mol}$ and $u_{P S}=-2875 \mathrm{~J} / \mathrm{mol}$, and estimate $L_{M}=3.494 \times 10^{4} \mathrm{~J} / \mathrm{mol}$ and $L_{P}=3.419 \times 10^{4} \mathrm{~J} / \mathrm{mol}$ from vapor pressure data. ${ }^{53}$ The interlayer distance is taken to be $6.435 \AA$.

For a given $\Delta T$ we then numerically minimize the excess free energy per unit area as a function of both the concentrations $x_{k}$ and the number of layers $n$. We obtain profiles that show clear phase separation qualitatively similar to the density profiles shown in Fig. 2 . The crossover from one of the coexisting concentrations to the other can be fitted well with an error function profile for thicker films (or small $\Delta T$ ). For a bulk interface $\left(u_{i S}=\Delta T=\Delta h=0, n \geqslant 30\right)$ we find $w_{0}$ $=16.15 \AA$ in decent agreement with the value of $18.6 \AA$ derived above from ellipsometry measurements. For the confined interface we estimate its intrinsic width from the maximum slope of the profile. The result is shown as the filled squares in Fig. 3. The jaggedness arises from the discrete number of layers in the lattice model. For a finer mesh of temperature differentials, $\Delta T$, the dependence of width on $d_{1}$ would exhibit discrete steps. Substrate and liquid/vapor

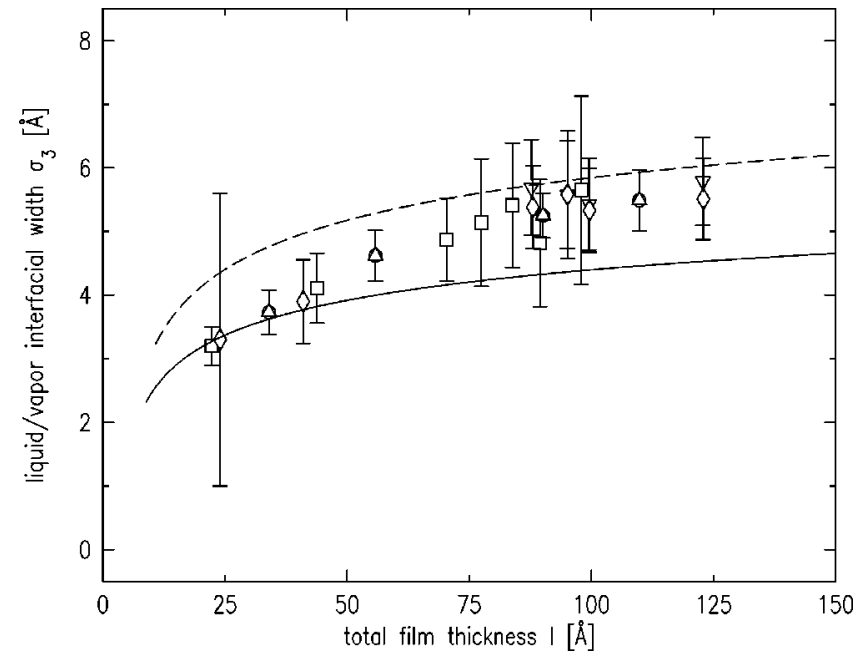

FIG. 6. Width of the liquid/vapor interface $\left(\sigma_{3}\right)$ as a function of total film thickness from synchrotron data from three separate fillings. The dashed line shows predictions from Eq. (7) with $\gamma$ $=12.5 \mathrm{mN} / \mathrm{m}$ (bulk PF), while the solid line uses $\gamma=23.15 \mathrm{mN} / \mathrm{m}$ (bulk MC). Both cases use $w_{0}=0$. See text for discussion.

interfacial roughness are neglected in this model, and for a small number of layers $\left(n \leqslant 6\right.$ or $\left.d_{1} \leqslant 23 \AA\right)$ the resulting profiles no longer justify the concept of an interface between two coexisting phases. For $n \geqslant 7$ the calculations show a reduction in $w_{0}$ by up to a factor of 2 relative to $w_{0}(\infty)$. It is also interesting to note that even at $d_{1} \approx 60-80 \AA$ $\geqslant 4 w_{0}(\infty)$ the width has still not reached its predicted bulk value. Finally the crosses show the convolution of the estimate for the intrinsic width with the reduced $\mathrm{CW}$ roughness derived in the Appendix.

As a result of the large scatter and uncertainty in the individual measured values for $\sigma_{2}$ it is difficult to extract an accurate value for the capillary wave contribution. Nevertheless one can see that interfacial fluctuations are strongly or perhaps even almost completely suppressed. If, for example, we assume based on the regular solution model that at $d_{1}$ $=45 \AA w_{0} \approx 11 \AA$ and the measured total interfacial width $\sigma_{2} \approx 13 \AA$, the CW roughness would have to be only $7 \AA$, compared to the value of $21 \AA$ predicted by Eq. (5). This value could be achieved, for example, by setting $\gamma$ $\approx 7 \mathrm{mN} / \mathrm{m}$; however, this corresponds to a 12 -fold increase relative to the macroscopic surface tension.

\section{The liquid/vapor interface}

In Fig. 6 we show the best fit results for the width of the liquid/vapor interface, $\sigma_{3}$, from synchrotron data, together with theoretical predictions according to Eqs. (7) and (13), as a function of total film thickness $l=d_{1}+d_{2}$. The solid and dashed lines use the bulk surface tensions for pure MC and $\mathrm{PF}$, respectively.

In contrast to the confined interface we find the roughness of the free interface close to theoretical predictions. As expected it also increases slowly with increasing film thickness. For thinner films $\sigma_{3}$ lies close to the values expected for pure MC. For thicker films it gradually moves closer to values for pure $\mathrm{PF}$ at the free surface. 


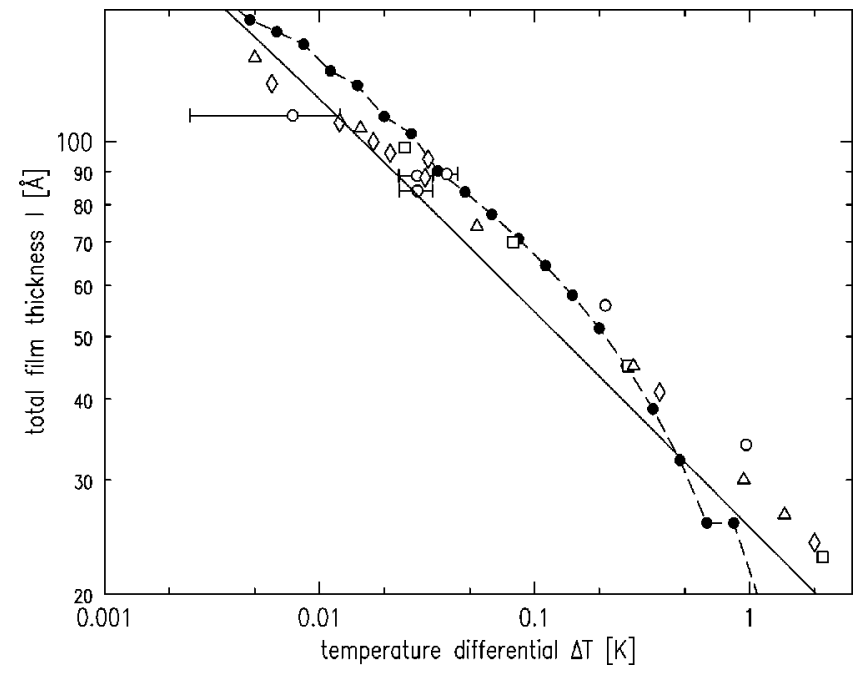

FIG. 7. Total film thickness $l$ versus temperature offset $\Delta T$. Open symbols are from reflectivity data. For clarity only data from one filling (open circles) is shown with error bars. Solid circles are results from the regular solution model. The solid line is the prediction from the simple model of Eq. (12).

Ignoring the large uncertainties in the values for $\sigma_{3}$ the data could therefore be interpreted as follows: the thinnest films cannot support the formation of two separate MC- and PF-rich layers and are dominated by the preferred adsorption of $\mathrm{MC}$ at the substrate, leading to a smaller $\mathrm{CW}$ roughness due to the larger surface tension of MC. For thicker films phase separation becomes possible, resulting in a gradual reduction in the surface tension (and thus an increase in $\mathrm{CW}$ roughness) due to the increased PF concentration at the free surface. The roughness remains slightly below the values predicted for a composite film with pure PF at the free surface. This interpretation is entirely consistent with the previously discussed density profiles. Alternatively it will be interesting to investigate the apparent slight decrease in the surface width for thinner films relative to theoretical curves with more precise experiments on monocomponent films.

\section{Wetting behavior}

As outlined in Sec. III the dependence of film thickness versus $\Delta T$ is a good probe for testing the interaction between substrate and molecules in the adsorbed film ${ }^{24,54}$ and to verify complete wetting.

Figure 7 shows the total wetting film thickness $l$ as a function of temperature differential $\Delta T$ for data from four separate fillings of the sample cell. Experiments usually start with a large temperature offset and films are measured at gradually increasing thicknesses until $\Delta T$ becomes so small that temperature fluctuations lead to instabilities in thickness. The offset $\Delta T$ is then increased and thinner films are measured again. Although there are no systematic differences between the film profiles measured in the cooling and heating cycles we do find that for the same $\Delta T$ films remain slightly thicker on cooling (e.g., 94 vs $88 \AA$ at $\Delta T$ $=32 \mathrm{mK}$ ). This might be due to a very large time constant as part of the wetting/phase separation process or due to some very weak thermal links in the wetting cell.
The solid line in Fig. 7 shows the predictions from Eq. (12). For all but the thickest films one sees that the simple model outlined above somewhat underestimates the film thickness. This is not too surprising, since the model neglects both the effects of mixing and the fluctuations at the liquid/ liquid interface. The regular solution model used to estimate the intrinsic width takes the entropy of mixing into account. Its results are also shown and give good agreement with the data in an intermediate $\Delta T$ range; however, there are discrepancies at both small and large $\Delta T$. In view of the fact that the regular solution model does not take into account the effects of differing molecular densities, heat of mixing, capillary wave, and substrate roughness, or any other type of substrate inhomogeneities, the discrepancies are not particularly surprising.

Both the regular solution model and the simpler one based on only van der Waals potentials predict the observed dependence of the film thicknesses on temperature offset between substrate and solution. The fact that the observed thicknesses are mostly larger than the values predicted by the latter model suggests chemical mixing and/or interfacial fluctuations induce some film thickening. The smaller observed film thicknesses at the smallest $\Delta T$ values could be due to experimental factors such as small temperature gradients within the cell, substrate inhomogeneities, or insufficient sample purity. In summary the observed van der Waals effects of wetting by a composite film are of the type predicted by Evans and co-workers. $^{22}$

\section{E. Results at $T=60^{\circ} \mathrm{C}$}

Measurements were taken up to $T=60^{\circ} \mathrm{C}$, i.e., about $14{ }^{\circ} \mathrm{C}$ above the consolute temperature. At this temperature the bulk correlation length is expected to be $20 \AA$. Interpretation of the data is more difficult due to reduced electron density contrast within the films and apparently rougher or more diffuse interfaces, which leads to less certainty in the model parameters. Rotating anode data taken at 40, 45, 50, and $60^{\circ} \mathrm{C}$ show a gradual reduction in the amplitude of the interference fringes, indicative of a decreasing density difference $\phi_{2}-\phi_{1}$ with increasing temperature. We find no obvious behavior in our data that would point towards a transition from two-phase coexistence to a single-phase wetting film.

At $60^{\circ} \mathrm{C}$ the reflectivities generally still show the same qualitative behavior as below $T_{c}$, in that the first maximum in $R / R_{F}$ (for $q_{z}>q_{c}$ ) exceeds 1 . The example in Fig. 4 shows a maximum close to $q_{z}=0.12 \AA^{-1}$ that is barely greater than 1 . However, without the reduction in reflected intensity due to the finite roughness at the interfaces, $R / R_{F}$ would exceed 1 significantly. Fitting this data therefore also requires a nonmonotonic density profile. However, we can not achieve good fits with the present simple two-layer model over the full range of $q_{z}$ accessible at the synchrotron. Our data would be consistent with the much smaller density difference of $\phi_{2}-\phi_{1} \approx 0.1$ if it were only fitted to the lower $q_{z}$ region (similar to the range often available for neutron reflectivity ${ }^{55}$ ). Furthermore, if better data could be taken, interpretation would necessitate more sophisticated approaches 
such as density functional theories that explicitly take into account critical adsorption. It would be interesting to investigate the effects of confinement on critical adsorption upon approaching the consolute temperature from above. In thick enough films interfacial widths between preferentially adsorbed layers and a bulklike phase would be expected to scale with the bulk correlation length $\xi$ and to diverge at $T_{c},{ }^{6}$ but in a thin film geometry critical fluctuations should be suppressed in a way similar to the $\mathrm{CW}$ roughness and intrinsic width at $30^{\circ} \mathrm{C}$.

\section{DISCUSSION}

Our x-ray reflectivity data clearly show evidence of phase separation in thin wetting films from a binary vapor below the upper critical solution temperature of the bulk mixture. Very good agreement is obtained between the data and the simplest conceivable density profile model consisting of only two layers of constant electron density for the adsorbed fluid and a $\mathrm{Si}$ substrate. Alternative models, such as those that include a native oxide layer on the Si substrate or with different representations of the profile leave results for total film thickness and roughnesses basically unchanged relative to their error bars, while layer densities differ by no more than $10 \%$ from the two-layer model.

Regardless of uncertainties at $30^{\circ} \mathrm{C}$ the density of the liquid in contact with the substrate lies between pure MC and the value for the bulk MC-rich phase, while the difference $\phi_{2}-\phi_{1}$ within the wetting films is slightly smaller than that for the coexisting bulk phases. As seen in Fig. 2 we also find the electron density of the PF-rich layer to be somewhat (about 13\%) below the predicted bulk value. Forcing $\phi_{2}$ close to its bulk value makes the fits unacceptable. The introduction of a monolayer of pure PF at the free surface also leads to much poorer fits. This is in contrast to density profiles used to model recent neutron reflectivity data from the free surface of a bulk mixture of PF/MC. ${ }^{56}$ However, it is not clear whether or not one should necessarily expect that the density of the wetting films should be identical to that of the bulk mixture. For example, the relative excess volume of mixing in bulk PF/MC has been found to be on the order of 2-3\% just below $T_{c},{ }^{57}$ which leads to a corresponding reduction in electron density that has been neglected in our analysis. The observed density at the liquid/vapor interface could therefore be due to a combination of reduced molecular number density and higher MC concentration. Future measurements on wetting films from vapors of either PF or MC might be able to illuminate this point. ${ }^{58}$

Once the films become thinner than $l \approx 40 \AA$ the experiment does not show unambiguous evidence for two regions of different uniform density. The competition between opposing preferred adsorption at the confining walls and minimization of the concentration gradient thus seems to prevent the formation of separate phases parallel to the walls. Interestingly, this also is predicted by the regular solution model.

The liquid/liquid interface is positioned close to the center of the composite wetting film as would be expected for a system with antisymmetric boundary conditions as realized through the Hamaker constants of the present system. The measured interfacial width $\sigma_{2}$ shows a weak dependence on film thickness. The width is expected to arise from a convolution between a mean field intrinsic width and thermal CW roughening. Although long-range dispersion interactions with the substrate reduce the $\mathrm{CW}$ roughness and introduce a distance dependence according to Eqs. (5) and (6), they do not reduce it enough to agree with the measured values for the liquid/liquid interface. CW roughness alone already exceeds the observed widths. On the other hand basically the same value for the intrinsic width is obtained from both the measured correlation lengths of the bulk phases and the regular solution model. The regular solution model also displays a significant reduction in intrinsic width with decreasing film thickness and shows how confinement leads to sharper interfaces in the absence of capillary waves. Interestingly, the values for the intrinsic width are very close to the full measured widths. In view of this it is not likely that possible errors in the Hamaker constants could account for the discrepancies. For example, since we estimate Hamaker constants from bulk values of the pure phases, the differences between the interactions between the MC- and PF-rich phases should actually be smaller than the values used here. Correction for this effect would lead to a reduction of $A_{\text {eff }}$ in Eq. (10) and a CW roughness even greater than shown in Fig. 3. Therefore we are forced to conclude that the $\mathrm{CW}$ contribution is much smaller than predicted. Its weak dependence on $A_{\text {eff }}$ makes it more plausible that an effective increase of the interfacial stiffness $\gamma$ in the confined system leads to reduced $\mathrm{CW}$ roughness. There still is no quantitative explanation for such an increase in $\gamma$. In contrast we find the width of the free surface dominated by $\mathrm{CW}$ roughness in reasonable agreement with estimates from the simple Hamaker approach.

The PF/MC system differs in several respects from thin films of binary mixtures of long-chain polymers. The PF/MC molecules can be considered almost spherical and nonpolar and are much smaller than the polymers. Their simple interactions should be much easier to estimate and simulate via Monte Carlo methods. Our experiment therefore offers a different physical system that also displays reduced interfacial width in a confined geometry. Our results confirm that the suppression of $\mathrm{CW}$ roughness and intrinsic width is a general effect due to geometric factors only and not specific to certain types of molecules or details of intermolecular interactions. A further difference lies in the fact that the polymer films typically are spin cast onto their substrates, which predetermines the number of molecules of each species in the films (canonical ensemble). In our experiment the wetting films are in contact with their relatively high-pressure vapor phase, allowing for very fast exchange of particles (grand canonical ensemble) and equilibration, and making the wetting film thickness a physically meaningful parameter in the description of the wetting process.

Theoretical models for thin films between competing walls usually assume perfectly antisymmetric interactions with the walls. While this condition is approximately realized in our system, the relative strengths of interactions with the "walls" could in principle be fine-tuned through modification of the substrate surface (e.g. coverage with self- 
assembled monolayer or Langmuir-Blodgett films of specifically selected molecules) from the perfectly antisymmetric to the symmetric case. However, most theoretical models so far have only treated short-range interactions with the substrate. For example, it is still questionable whether an interface localization transition ${ }^{59,60}$ exists in real systems that are much better described by long-range van der Waals-type interactions. Our experimental geometry and technique could be used to search for such a transition in a suitable mixture.

Our results also show that $\mathrm{x}$-ray reflectivity is an excellent tool for the investigation of wetting phenomena in more complex systems such as binary mixtures. The very good electron density contrast between the two components in mixtures of hydrocarbons and perfluorocarbons combined with the high photon flux available at synchrotron sources allows ångstrom-type resolution in the derived density profiles. Future experiments might be improved through additional sample purification, and better substrate characterization could remove remaining small uncertainties in the density profiles. Of course it will also be interesting to employ more sophisticated density functional models in the description of our data.

The current experiment can be extended in numerous ways. More extensive measurements at different temperatures above and below $T_{c}$ are in order. Assuming that the two components do not freeze first, at least two wetting transitions are expected at temperatures below $30{ }^{\circ} \mathrm{C} .{ }^{39}$ At low temperatures the substrate should be dry, and the substrate/ vapor interface should be wetted by the MC-rich phase upon increasing the temperature above some value. At somewhat higher temperatures $\left[T \geqslant 6-8{ }^{\circ} \mathrm{C}\right.$ (Ref. 56)] the MC-rich/ vapor interface will then be wetted by a PF-rich layer, leading to the composite film structure observed at $30^{\circ} \mathrm{C}$. Even more complicated scenarios have been discussed previously. ${ }^{22}$ Different concentrations in the liquid reservoir and therefore in the vapor phase would allow us to control the composition and thereby the position of the liquid/liquid interface within a wetting film of a given thickness. In this way the middle interface could be moved farther away from the substrate. A different geometry that would enable us to follow the development of density profiles and interfacial fluctuations to thick films, and thus to bulk behavior, would be to lower a horizontal substrate below the surface of the bulk mixture. Measurements performed in this way could be compared with previous ellipsometry, ${ }^{17-19,40}$ evanescentwave-generated fluorescence spectroscopy, ${ }^{20}$ neutron, ${ }^{55,56}$ and x-ray scattering experiments ${ }^{42,61}$ on PF/MC and related mixtures.

\section{SUMMARY}

We have investigated wetting of a $\mathrm{Si}$ substrate by the vapor of a binary mixture of methylcyclohexane and perfluoromethylcyclohexane with $\mathrm{x}$-ray reflectivity. $16^{\circ} \mathrm{C}$ below the consolute temperature we find composite wetting films made up of an MC-rich phase at the substrate and a PF-rich phase at the liquid/vapor interface. The interface between the two phases stays close to the middle of the composite film for total thicknesses between 40 and $135 \AA$, while thinner films cannot support two separate identifiable phases. This system can be viewed as a realization of a binary system confined between walls with competing interactions. The width of the liquid/liquid interface depends weakly on the distance from the substrate. It is considerably smaller than capillary wave theory and mean field estimates of the intrinsic width predict, similar to previous observations on spin cast films from binary mixtures of long-chain polymers. Geometric confinement therefore reduces thermal capillary waves in a manner compatible with an effective increase in interfacial stiffness, and at the same time reduces the intrinsic width of the confined interface. Measurements above $T_{c}$ still show signs of preferred adsorption at the confining walls.

\section{ACKNOWLEDGMENTS}

We wish to acknowledge S. Coburn, J. P. Hill, B. Ocko, and E. DiMasi for their technical support at beamline X22A of the NSLS. This work was supported through NSF Grant No. NSF-DMR-98-72817. Brookhaven National Laboratory was supported by DOE Grant No. DE-AC02-76CH00016.

\section{APPENDIX: CAPILLARY WAVE ROUGHNESS OF THE CONFINED LIQUID/LIQUID INTERFACE}

The capillary wave roughness $\sigma_{\mathrm{CW}}=\sqrt{\left\langle u^{2}(0)\right\rangle}$ of the MC-rich/PF-rich interface discussed in Sec. III B is based on the interfacial tension $\gamma$ and the leading $u^{2}$ term of the power-series expansion for the van der Waals potential $\delta G(u)=G(u)-G(0)$ [see Eqs. (5)-(10)]. For thin films the roughness $\sigma_{\mathrm{CW}}$ obtained in this way becomes comparable to the film thickness, which is clearly not physically plausible. This problem arises because limiting the power-series expansion only to the $u^{2}$ term does not account for the fact that the full potential $\delta G(u)$ diverges as $u \rightarrow d_{1}$ or $d_{2}$. In this appendix we attempt to estimate the confinement effects on $\sigma_{\mathrm{CW}}$ arising from reduced film thicknesses by taking into account the higher-order terms in $\delta G(u)$.

In what follows, we assume that (i) the equilibrium film thicknesses of the MC-rich and PF-rich layers are equal $\left(d_{1}=d_{2}\right)$; (ii) the total film thickness $l=d_{1}+d_{2}=2 d_{1}$ is fixed, which is equivalent to neglecting the effect of the free liquid/vapor interfacial roughness $\sigma_{3}$; and (iii) the Hamaker constants satisfy the relation

$$
A_{S M}-A_{M M} \approx A_{S P}-A_{P P} .
$$

As discussed already, assumption (i) is supported by both the theoretical expectations and the experimental results presented in Secs. III and IV. Assumption (ii) is consistent with the fact that for the present system fluctuations of the buried interface are essentially decoupled from those of the free surface. Assumption (iii) implies that for each of the two molecular components, the difference between van der Waals interactions with the substrate and with itself is roughly the same. Based on the estimated values of $A_{i j}$ given in Table I, the two sides in Eq. (A1) agree within $15 \%$ for the $\mathrm{Si} / \mathrm{MC} / \mathrm{PF}$ system. 
The application of these assumptions to Eq. (8) results in a symmetric form of the van der Waals potential per unit area for the height fluctuations $u$ of the liquid/liquid interface:

$$
\delta G(u)=\frac{1}{2} \frac{A_{\mathrm{eff}}}{12 \pi}\left[\frac{1}{\left(d_{1}+u\right)^{2}}+\frac{1}{\left(d_{1}-u\right)^{2}}-\frac{2}{d_{1}^{2}}\right],
$$

or by expanding as a power series,

$$
\delta G(u)=\frac{A_{\mathrm{eff}}}{12 \pi d_{1}^{2}} \sum_{n=1}^{\infty}(2 n+1)\left[\frac{u}{d_{1}}\right]^{2 n} .
$$

The effective Hamaker constant is given by

$$
A_{\text {eff }}=2\left[A_{S M}-A_{S P}+A_{M P}-A_{M M}\right]=2\left[A_{M P}-A_{P P}\right] .
$$

The leading term in Eq. (A3) is identical to Eq. (9). In the case of a free or internal interface of a bulk liquid (i.e., $d_{1}$ $\rightarrow \infty$ ), the term in the Hamiltonian that is most responsible for limiting the $\mathrm{CW}$ roughness is usually that of the interfacial tension. However, the divergence of $\delta G(u)$ at the confining walls that is explicit in Eq. (A2) implies that the correction to $\sigma_{\mathrm{CW}}$ due to the nonharmonic terms of the longrange van der Waals potential should become increasingly important as $d_{1}$ decreases towards the bulk value of $\sigma_{\mathrm{CW}}$. Retaining the higher-order terms in $\delta G(u)$, the thermal average of total energy per area $A$ of the liquid/liquid interface is given by

$$
\begin{aligned}
\frac{\langle U\rangle}{A} & =\frac{1}{2} \gamma\left\langle|\vec{\nabla} u(\vec{r})|^{2}\right\rangle+\langle\delta G(u)\rangle \\
& =\frac{1}{2} \gamma\left\langle|\vec{\nabla} u(\vec{r})|^{2}\right\rangle+\frac{A_{\text {eff }}}{12 \pi d_{1}^{2}} \sum_{n=1}^{\infty}(2 n+1) \frac{\left\langle u^{2 n}(\vec{r})\right\rangle}{d_{1}^{2 n}},
\end{aligned}
$$

where $\vec{r}=\vec{r}_{x y}$. Expanding the height fluctuations $u=u(r)$ as the sum of allowed $\mathrm{CW}$ modes, i.e.,

$$
u(\vec{r})=\frac{1}{(2 \pi)^{2}} \int_{q<q_{\max }} d^{2} \vec{q} \tilde{u}(\vec{q}) \exp (-i \vec{q} \cdot \vec{r}),
$$

where $\vec{q}=\vec{q}_{x y}$, the various expectation values that appear in Eq. (A5) can be expressed as

$$
\begin{gathered}
\left\langle|\vec{\nabla} u(\vec{r})|^{2}\right\rangle=\frac{1}{(2 \pi)^{2}} \int d^{2} \vec{q} \frac{1}{A}\left\langle|\tilde{u}(\vec{q})|^{2}\right\rangle q^{2}, \\
\left\langle u^{2}(\vec{r})\right\rangle=\frac{1}{(2 \pi)^{2}} \int d^{2} \vec{q} \frac{1}{A}\left\langle|\tilde{u}(\vec{q})|^{2}\right\rangle, \\
\left\langle u^{2 n}(\vec{r})\right\rangle=\left[\frac{1}{(2 \pi)^{2}}\right]^{2 n}\left\langle\int d^{2} \vec{q}_{1} \cdots d^{2} \vec{q}_{2 n} \tilde{u}\left(\vec{q}_{1}\right) \cdots \tilde{u}\left(\vec{q}_{2 n}\right)\right. \\
\left.\quad \times \exp \left[-i\left(\vec{q}_{1}+\cdots+\vec{q}_{2 n}\right) \cdot \vec{r}\right]\right\rangle
\end{gathered}
$$

$$
\begin{aligned}
= & {\left[\frac{1}{(2 \pi)^{2}}\right]^{2 n-1} \int d^{2} \vec{q}_{1} \cdots d^{2} \vec{q}_{2 n} } \\
& \times \frac{1}{A}\left\langle\tilde{u}\left(\vec{q}_{1}\right) \cdots \tilde{u}\left(\vec{q}_{2 n}\right)\right\rangle \delta\left(\vec{q}_{1}+\cdots+\vec{q}_{2 n}\right) .
\end{aligned}
$$

Equations (A7) and (A9) are based on the fact that the expectation values should be independent of the position $r\left[\left\langle u^{2}(r)\right\rangle=\left\langle u^{2}(0)\right\rangle\right.$, etc. $]$ and that $u(r)$ is real $\left[\tilde{u}^{*}(\vec{q})\right.$ $=\tilde{u}(-\vec{q})]$.

Note that for $n \geqslant 2$ each expression $\left\langle u^{2 n}(r)\right\rangle$ in Eq. (A9) consists of a multiple sum over the surface capillary modes and is more difficult to evaluate than the terms in Eq. (A7). In order to simplify the computation, we make the assumption that the average in Eq. (A8) can be approximated as the product of two separate averages, as follows:

$$
\begin{aligned}
\left\langle u^{2 n}(\vec{r})\right\rangle \approx & {\left[\frac{1}{(2 \pi)^{2}}\right]^{2 n} \sum_{i=1}^{2 n} \sum_{j>i}^{2 n}\left\langle\int d^{2} \vec{q}_{i}\right.} \\
& \left.\times \int d^{2} \vec{q}_{j} \tilde{u}\left(\vec{q}_{i}\right) \tilde{u}\left(\vec{q}_{j}\right) \exp \left[-i\left(\vec{q}_{i}+\vec{q}_{j}\right) \cdot \vec{r}\right]\right\rangle \\
& \times\left\langle\prod_{k \neq i, j}^{2 n} \int d^{2} \vec{q}_{k} \tilde{u}\left(\vec{q}_{k}\right) \exp \left(-i \vec{q}_{k} \cdot \vec{r}\right)\right\rangle .
\end{aligned}
$$

By applying Eq. (A9) to each average in Eq. (A10) and noting that each term in the double sum contributes equally, the above can be reexpressed as

$$
\left\langle u^{2 n}(\vec{r})\right\rangle \approx n(2 n-1)\left\langle u^{2(n-1)}(\vec{r})\right\rangle \frac{1}{(2 \pi)^{2}} \int d^{2} \vec{q} \frac{1}{A}\left\langle|\widetilde{u}(\vec{q})|^{2}\right\rangle .
$$

This is exactly the recursion relation between $\left\langle u^{2 n}\right\rangle$ and $\left\langle u^{2(n-1)}\right\rangle$ that is obtained when $\{\tilde{u}(\vec{q})\}$ obeys Gaussian statistics. Note that Eq. (A11) gives the correct expression for the leading term $(n=1)$ shown in Eq. (A7).

By substituting Eq. (A7) and (A11) into (A5) and integrating over total area $A$, the average total energy $\langle U\rangle$ can be expressed as a single sum of square amplitudes of allowed CW modes:

$$
\begin{aligned}
\langle U\rangle & \approx \frac{1}{(2 \pi)^{2}} \int_{q<q_{\max }} d^{2} \vec{q} \frac{1}{2}\left[b_{\mathrm{eff}}+\gamma q^{2}\right]\left\langle|\tilde{u}(\vec{q})|^{2}\right\rangle \\
& =\sum_{\vec{q}} \frac{1}{2}\left[b_{\mathrm{eff}}+\gamma q^{2}\right] \frac{1}{A}\left\langle|\tilde{u}(\vec{q})|^{2}\right\rangle,
\end{aligned}
$$

where the "effective" prefactor for the harmonic term $b_{\text {eff }}$ is given by

$$
b_{\mathrm{eff}} \equiv \frac{A_{\mathrm{eff}}}{12 \pi d_{1}^{4}} \sum_{n=1}^{\infty}(2 n+1)(2 n)(2 n-1) \frac{\left\langle u^{2(n-1)}\right\rangle}{d_{1}^{2(n-1)}},
$$

and $\left\langle u^{2(n-1)}\right\rangle=\left\langle u^{2(n-1)}(r)\right\rangle=\left\langle u^{2(n-1)}(0)\right\rangle$. Finally, an expression for the $\mathrm{CW}$ roughness $\sigma_{\mathrm{CW}}=\sqrt{\left\langle u^{2}(0)\right\rangle}$ in terms of $b_{\text {eff }}$ can be obtained by the application of the equipartition 
theorem to Eq. (A12) and the summing of the resulting mean-square amplitudes of allowed modes [see Eq. (5)]:

$$
\sigma_{\mathrm{CW}}^{2}=\left\langle u^{2}\right\rangle=\frac{k_{B} T}{4 \pi \gamma} \ln \left[1+\frac{\gamma q_{\mathrm{max}}^{2}}{b_{\mathrm{eff}}}\right] .
$$

Note that the long-wavelength cutoff is equal to $\xi_{\|}$ $=\sqrt{\gamma / b_{\text {eff }}}$, which reduces to Eq. (6) if $b_{\text {eff }}$ is replaced by its leading term in Eq. (A13). We have assumed that $\xi_{\|}^{-1}$ is much greater than $q_{\min }$ but could be comparable to $q_{\max }$ $\approx 2 \pi / B_{0}$. The latter takes into account the possibility $\xi_{\|}$ $\approx B_{0}$.

It is clear from Eqs. (A13) and (A14) that the estimates for the confinement effect on CW roughness depend on how the terms $\left\langle u^{2(n-1)}\right\rangle$ in the sum for $b_{\text {eff }}$ are evaluated. The following presents two out of many possible approximation methods for evaluation of $\left\langle u^{2(n-1)}\right\rangle$ and therefore the calculation of $\left\langle u^{2}\right\rangle$.

\section{Self-consistent $\left\langle u^{2}\right\rangle$ via Gaussian approximation for $\left\langle u^{2(n-1)}\right\rangle$}

It can be shown that if a fluctuating variable $x$ obeys Gaussian statistics, the higher-order moments are related to the mean-square average through $\left\langle x^{2 n}\right\rangle=(2 n-1) ! !\left\langle x^{2}\right\rangle^{n}$. Therefore, if the height fluctuations $u$ of the confined liquid/ liquid interface were to follow a Gaussian distribution, the substitution of $\left\langle u^{2(n-1)}\right\rangle \approx(2 n-3) ! !\left\langle u^{2}\right\rangle^{n-1}$ in Eq. (A13) could be approximated by

$$
b_{\mathrm{eff}, G}(N)=\frac{A_{\mathrm{eff}}}{6 \pi d_{1}^{4}} \sum_{n=1}^{N} n(2 n+1) ! !\left[\frac{\left\langle u^{2}\right\rangle}{d_{1}^{2}}\right]^{n-1} .
$$

If this Gaussian approximation were valid, both sides of Eq. (A14) would depend on the mean-square roughness $\left\langle u^{2}\right\rangle$ and, for a given $N$ in Eq. (A15), one would be able to solve Eq. (A14) for $\left\langle u^{2}\right\rangle$ self-consistently. A final solution would result if the self-consistent solution for $\left\langle u^{2}\right\rangle$ were to converge as $N \rightarrow \infty$. Similar procedures are often invoked to justify the random phase approximation in statistical mechanics.

Unfortunately, the power series for $b_{\text {eff, } G}(N)$ in Eq. (A15) does not converge as $N \rightarrow \infty$ for any nonzero value of $\left\langle u^{2}\right\rangle / d_{1}^{2}$. As a result, the only self-consistent solution for $N$ $\rightarrow \infty$ is $\left\langle u^{2}\right\rangle=0$. If this divergence of $b_{\mathrm{eff}, G}$ were a physical consequence of the confining potential $\delta G(u)$ given in Eqs. (A2) and (A3), it would imply that the $\mathrm{CW}$ roughness $\sigma_{\mathrm{CW}}$ $=\sqrt{\left\langle u^{2}\right\rangle}$ of the confined liquid/liquid interface would have to be completely suppressed, i.e., $\sigma_{\mathrm{CW}}=0$. In fact the effect arises because the Gaussian approximation implicitly requires a parabolic potential that places no restrictions on the magnitude of possible fluctuations of $u$. It is clear that the divergence in the full van der Waals potential $\delta G(u)$ as $u$ $\rightarrow d_{1}$ confines $\left\langle u^{2(n-1)}\right\rangle$ in a way that the parabolic potential does not. As a result the Gaussian approximation overestimates $b_{\text {eff }}$, which, in turn, underestimates the value of $\sigma_{\mathrm{CW}}$.

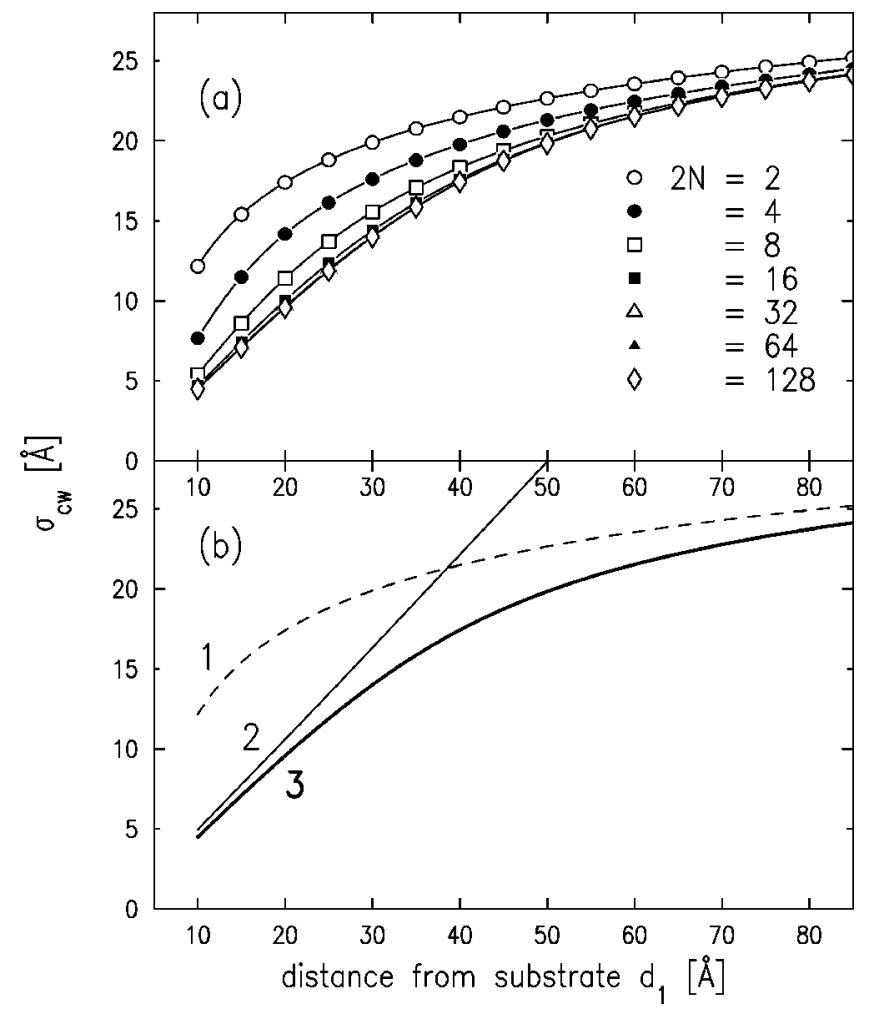

FIG. 8. Calculated CW roughness $\sigma_{\mathrm{CW}}=\sqrt{\left\langle u^{2}\right\rangle}$ of the MC-rich/ PF-rich interface, as a function of the distance $d_{1}$ from the substrate. (a) $\sigma_{\mathrm{CW}}$ based on self-consistent solutions for $\left\langle u^{2}\right\rangle$ using Eqs. (A14) and (A19) with the approximations (A17) and (A18) for $\left\langle u^{2(n-1)}\right\rangle$ in the factor $b_{\text {eff }}$. The curves in (a) have been obtained by retaining all the terms up to a highest term given by $u^{2 N}$ in the power-series expansion of $\delta G(u)$ [Eq. (A3)], where the value of $2 N$ for each curve is indicated. The values of physical parameters used in the calculations of all the curves in (a) are given by $T$ $=30^{\circ} \mathrm{C}, \quad \gamma=0.58 \mathrm{mN} / \mathrm{m}, A_{\text {eff }}=3.3 \times 10^{-20} \mathrm{~J}$, and $q_{\max }=2 \pi / B_{0}$, where $B_{0}=6.0 \AA$. (b) curve $1: \gamma=0.58 \mathrm{mN} / \mathrm{m}, \delta G(u) \propto u^{2}$, same as the $2 N=2$ curve in (a) (dashed line); curve 2: $\gamma=0$, full $\delta G(u)$, calculated using Eq. (A16) (thin solid line); Curve 3: convergent solution from self-consistent $\left\langle u^{2}\right\rangle$ in (a) (thick solid line).

\section{Self-consistent $\left\langle u^{2}\right\rangle$ via approximation $\left\langle u^{2(n-1)}\right\rangle$ $\approx\left[\left\langle u^{2(n-1)}\right\rangle /\left\langle u^{2}\right\rangle^{n-1}\right]_{\gamma=0}\left\langle u^{2}\right\rangle^{n-1}$}

The evaluation of expectation values would be simple if the energy density $V$ (per area in the present case) depended only on the fluctuating field $u(r)$ and not on its derivatives. Taking $\gamma \approx 0$, the moments $\left\langle u^{2 m}\right\rangle_{\gamma=0}$ for the CW fluctuations can be expressed as

$$
\left\langle u^{2 m}\right\rangle_{\gamma=0}=\frac{\int_{0}^{d_{1}} d u u^{2 m} \exp \left(-\frac{4 \pi}{q_{\max }^{2}} \frac{\delta G(u)}{k_{B} T}\right)}{\int_{0}^{d_{1}} d u \exp \left(-\frac{4 \pi}{q_{\max }^{2}} \frac{\delta G(u)}{k_{B} T}\right)},
$$

where the van der Waals potential $\delta G(u)$ is given by Eq. (A2). The factor $4 \pi / q_{\max }^{2}$ in the above equation defines the area in phase space for one surface mode. This factor can be obtained by considering the case of a simple harmonic potential $V(u)=(1 / 2) b u^{2}$, which leads to $\left\langle u^{2}\right\rangle$ 
$=k_{B} T q_{\max }^{2} /(4 \pi b)$ [take the limit $\gamma \rightarrow 0$ in Eq. (A14)], and using the fact that the Gaussian statistics implied by this potential require that each exponent in Eq. (A16) be equal to $u^{2} /\left(2\left\langle u^{2}\right\rangle\right)$.

Recall that the divergence of $b_{\text {eff, } G}$ encountered in the Gaussian approximation results from the fact that the approximation $\left\langle u^{2(n-1)}\right\rangle \approx(2 n-3) ! !\left\langle u^{2}\right\rangle^{n-1}$ overestimates $\left\langle u^{2(n-1)}\right\rangle$ due to the implicit use of the weaker parabolic potential that allows unbounded variations of $u$. On the other hand, the full potential $\delta G(u)$ in Eq. (A16) limits large fluctuations in $u$. Therefore, a more reasonable approximation for $\left\langle u^{2(n-1)}\right\rangle$ in $b_{\text {eff }}$ is (i) to preserve the relation $\left\langle u^{2(n-1)}\right\rangle$ $\propto\left\langle u^{2}\right\rangle^{n-1}$, but (ii) estimate the "proportionality constant" (i.e., the ratio $\left\langle u^{2(n-1)}\right\rangle /\left\langle u^{2}\right\rangle^{n-1}$ ) using the expectation values for the $\gamma=0$ case:

$$
\left\langle u^{2(n-1)}\right\rangle \approx g_{n-1}\left\langle u^{2}\right\rangle^{n-1},
$$

where the factor $g_{n-1}$ is defined as

$$
g_{n-1} \equiv \frac{\left\langle u^{2(n-1)}\right\rangle_{\gamma=0}}{\left\langle u^{2}\right\rangle_{\gamma=0}^{n-1}}
$$

and calculated numerically using Eq. (A16). By substituting Eq. (A17) into (A13), $b_{\text {eff }}$ can be approximated by

$b_{\mathrm{eff}}(N) \approx \frac{A_{\mathrm{eff}}}{12 \pi d_{1}^{4}} \sum_{n=1}^{N}(2 n+1)(2 n)(2 n-1) g_{n-1}\left[\frac{\left\langle u^{2}\right\rangle}{d_{1}^{2}}\right]^{n-1}$.

For any given $N$ in (A19), Eq. (A14) can be solved selfconsistently for $\left\langle u^{2}\right\rangle$ to obtain $\sigma_{\mathrm{CW}}(N)$. Figure 8(a) shows the results of self-consistent solutions at different $N$ for the $\mathrm{CW}$ roughness $\sigma_{\mathrm{CW}}=\sqrt{\left\langle u^{2}\right\rangle}$ of the confined MC-rich/PF-rich interface as a function of $d_{1}$. It is clear that the calculated curves converge with increasing $N$. Figure 8(b) compares the convergent result for $\sigma_{\mathrm{CW}}$ of confined films (curve 3) against the case of $\delta G(u) \propto u^{2}$ (i.e., $2 N=2$; curve 1), which can be considered as the extrapolation of the bulk behavior expected for thicker films with the same interfacial tension. Also shown in Fig. 8(b) is the roughness $\sqrt{\left\langle u^{2}\right\rangle_{\gamma=0}}$ (curve 2), calculated directly from Eq. (A16), that would be obtained if the interfacial tension were absent and the fluctuations in $u(r)$ were governed completely by the full van der Waals potential $\delta G(u)$.

Both the full potential $\delta G(u)$ and interfacial tension $\gamma$ contribute to the reduction of the $\mathrm{CW}$ roughness. Therefore, it is not surprising that curve 3, which takes into account both of these effects, lies below the other two curves in Fig. 8(b). A more important observation is that curve 3 displays the expected crossover between a $\gamma$-dominated regime at large $d_{1}$ and a $\delta G$-dominated regime at small $d_{1}$. Figure 8(b) shows that as the film thickness or $d_{1}$ increases, the $\mathrm{CW}$ roughness (curve 3) approaches the values expected from the unconfined, bulk behavior (curve 1). On the other hand, as $d_{1}$ decreases and becomes comparable to the bulk value for $\sigma_{\mathrm{CW}}$, non-negligible reduction in $\sigma_{\mathrm{CW}_{\circ}}$ expected from confinement becomes evident. For $d_{1}<20 \AA$, the $\mathrm{CW}$ roughness estimated here for the confined interface is smaller than the bulk value by a factor of 2 or more. The comparison between curves 2 and 3 shows that this reduction in $\sigma_{\mathrm{CW}}$ indeed originates from the dominance of the confining van der Waals potential at small $d_{1}$.

Despite the physical plausibility of these results, it is also important to note that the self-consistent $\mathrm{CW}$ roughness $\sigma_{\mathrm{CW}}=\sqrt{\left\langle u^{2}\right\rangle}$ estimated above is still too large to explain the small width experimentally observed for the MC-rich/PFrich interface. Curve 3 in Fig. 8(b), which only takes into account the $\mathrm{CW}$ roughness of the interface, still lies above the data points for the measured total width $\sigma_{2}$, to which both $\sigma_{\mathrm{CW}}$ and the intrinsic width $w_{0}$ contribute (see Fig. 3).
*Current address: Space Nanotechnology Laboratory, Center for Space Research, Massachusetts Institute of Technology, Cambridge, MA 02139.

${ }^{1}$ M. Schick, in Liquids at Interfaces, edited by J. Charvolin, J. F. Joanny, and J. Zinn-Justin (Elsevier Science, Amsterdam, 1990).

${ }^{2} \mathrm{~S}$. Dietrich, in Phase Transitions and Critical Phenomena, edited by C. Domb and J. L. Lebowitz (Academic, London, 1988), Vol. 12.

${ }^{3}$ R. Evans, J. Phys.: Condens. Matter 2, 8989 (1990).

${ }^{4}$ K. Binder, Adv. Polym. Sci. 138, 1 (1999).

${ }^{5}$ A. Maciolek, A. Ciach, and R. Evans, J. Chem. Phys. 108, 9765 (1998).

${ }^{6}$ M. E. Fisher and P. G. de Gennes, C. R. Adad. Sci. Paris B 287, 207 (1978).

${ }^{7}$ R. Cherrabi, A. Saout-Elhak, M. Benhamou, and M. Daoud, J. Chem. Phys. 111, 8174 (1999).

${ }^{8}$ M. Krech, J. Phys.: Condens. Matter 11, R391 (1999); N. B. Wilding and M. Krech, Phys. Rev. E 57, 5795 (1998).

${ }^{9}$ T. Kerle, J. Klein, and K. Binder, Phys. Rev. Lett. 77, 1318 (1996).

${ }^{10}$ M. Sferrazza, C. Xiao, R. A. L. Jones, D. G. Bucknall, J. Webster, and J. Penfold, Phys. Rev. Lett. 78, 3693 (1997).
${ }^{11}$ T. Kerle, J. Klein, and K. Binder, Eur. Phys. J. B 7, 401 (1999).

${ }^{12}$ K. Binder, M. Müller, F. Schmid, and A. Werner, J. Stat. Phys. 95, 1045 (1999).

${ }^{13}$ A. Werner, M. Müller, F. Schmid, and K. Binder, J. Chem. Phys. 107, 8175 (1997).

${ }^{14}$ M. Müller and K. Binder, Macromolecules 31, 8323 (1998).

${ }^{15}$ A. Werner, M. Müller, F. Schmid, and K. Binder, J. Chem. Phys. 110, 1221 (1999).

${ }^{16}$ D. E. L. Dyke, J. S. Rowlinson, and R. Thacker, Trans. Faraday Soc. 55, 903 (1959).

${ }^{17}$ R. B. Heady and J. W. Cahn, J. Chem. Phys. 58, 896 (1972).

${ }^{18}$ R. G. Howland, N.-C. Wong, and C. M. Knobler, J. Chem. Phys. 73, 522 (1980).

${ }^{19}$ J. W. Schmidt, Phys. Rev. A 41, 885 (1990).

${ }^{20}$ A.-M. Williamson and I. A. McLure, Physica A 234, 225 (1996).

${ }^{21}$ P. Wynblatt, A. Saúl, and D. Chatain, Acta Mater. 46, 2337 (1998).

${ }^{22}$ M. M. Telo da Gama and R. Evans, Mol. Phys. 48, 687 (1983); I. Hadjiagapiou and R. Evans, ibid. 54, 383 (1985).

${ }^{23}$ A recent study finds indirect evidence for phase separation in thin binary wetting films: A. Mukhopadhyay and B. M. Law, Phys. Rev. Lett. 83, 772 (1999). 
${ }^{24}$ I. M. Tidswell, T. A. Rabedeau, P. S. Pershan, and S. D. Kosowsky, Phys. Rev. Lett. 66, 2108 (1991); I. M. Tidswell, T. A. Rabedeau, P. S. Pershan, J. P. Folkers, M. V. Baker, and G. M. Whitesides, Phys. Rev. B 44, 10869 (1991).

${ }^{25}$ Although surfaces prepared in this way would be coated with a thin layer of native oxide, the measurements reported in this paper are not sensitive to the presence or absence of such a layer.

${ }^{26}$ S. K. Sinha, E. B. Sirota, S. Garoff, and H. B. Stanley, Phys. Rev. B 38, 2297 (1988).

${ }^{27}$ A. Braslau, P. S. Pershan, G. Swislow, B. M. Ocko, and J. AlsNielsen, Phys. Rev. A 38, 2457 (1988).

${ }^{28}$ M. K. Sanyal, S. K. Sinha, K. G. Huang, and B. M. Ocko, Phys. Rev. Lett. 66, 628 (1991).

${ }^{29}$ D. Andelman, J. F. Joanny, and M. O. Robbins, Europhys. Lett. 7, 731 (1988); M. O. Robbins, D. Andelman, and J. F. Joanny, Phys. Rev. A 43, 4344 (1991).

${ }^{30}$ J. Daillant and O. Belorgey, J. Chem. Phys. 97, 5824 (1992); 97, 5837 (1992).

${ }^{31}$ O. H. Seeck, P. Müller-Buschbaum, M. Tolan, and W. Press, Europhys. Lett. 29, 699 (1995).

${ }^{32}$ A. K. Doerr, M. Tolan, W. Prange, J.-P. Schlomka, T. Seydel, W. Press, D. Smilgies, and B. Struth, Phys. Rev. Lett. 83, 3470 (1999).

${ }^{33}$ P. S. Pershan, M. Fukuto, O. Gang, and R. K. Heilmann (unpublished).

${ }^{34}$ P. S. Pershan and J. Als-Nielsen, Phys. Rev. Lett. 52, 759 (1984).

${ }^{35}$ P. S. Pershan, M. Fukuto, O. Gang, and R. K. Heilmann (unpublished calculations).

${ }^{36}$ L. G. Parrat, Phys. Rev. 95, 359 (1954).

${ }^{37}$ L. Nevot and P. Croce, Rev. Phys. Appl. 15, 761 (1980).

${ }^{38}$ R. K. Heilmann and R. M. Suter, Phys. Rev. B 59, 3075 (1999).

${ }^{39}$ S. Dietrich and M. Schick, Phys. Rev. B 33, 4952 (1986).

${ }^{40}$ D. Ripple and C. Franck, Phys. Rev. A 44, 8289 (1991).

${ }^{41}$ S. Fisk and B. Widom, J. Chem. Phys. 50, 3219 (1969); J. Huang and W. W. Webb, ibid. 50, 3677 (1969).

${ }^{42}$ B. R. McClain, M. Yoon, J. D. Lister, and S. G. J. Mochrie, Eur. Phys. J. B 10, 45 (1999).

${ }^{43}$ A. J. Liu and M. E. Fisher, Physica A 156, 35 (1989).

${ }^{44}$ J. S. Rowlinson and B. Widom, Molecular Theory of Capillarity (Oxford University Press, Oxford, 1982).

${ }^{45} \mathrm{~J}$. Israelachvili, Intermolecular and Surface Forces, 2nd ed. (Academic, London, 1991).

${ }^{46}$ As shown by Tidswell et al. (Ref. 24) the effect of a thin native oxide layer makes very little difference in the wetting behavior.

${ }^{47}$ J. Israelachvili, Proc. R. Soc. London, Ser. A 331, 39 (1972).
${ }^{48}$ In principle, a thin oxide layer can change the wetting condition for very thin films; however, once the substrate is wetted the surface properties are not significantly changed by the presence or absence of oxide. This can be seen from Ref. 24; alternatively this can also be shown from a straightforward extension of Eqs. (8)-(10) to a model that includes an oxide layer.

${ }^{49}$ J. Daillant, L. Bosio, J. J. Benattar, and J. Meunier, Europhys. Lett. 8, 453 (1989); D. K. Schwartz, M. L. Schlossman, E. H. Kawamoto, G. J. Kellogg, P. S. Pershan, and B. M. Ocko, Phys. Rev. A 41, 5687 (1990); M. K. Sangal, S. K. Sinha, K. G. Huang, and B. M. Ocko, Ref. 28; W. Zhao et al., J. Chem. Phys. 97, 8536 (1992); M. Fukuto, R. K. Heilmann, P. S. Pershan, J. A. Griffiths, S. M. Yu, and D. A. Tirrell, Phys. Rev. Lett. 81, 3455 (1998).

${ }^{50}$ H. Tostmann, E. DiMasi, P. S. Pershan, B. M. Ocko, O. G. Shpyrko, and M. Deutsch, Phys. Rev. B 59, 783 (1999); E. DiMasi H. Tostmann, B. M. Ocko, P. S. Pershan, and M. Deutsch, ibid. 58, 13419 (1998); M. J. Regan, E. H. Kawamoto, S. Lee, P. S. Pershan, N. Maskil, M. Deutsch, O. M. Magnussen, B. M. Ocko, and L. E. Berman, Phys. Rev. Lett. 75, 2498 (1995).

${ }^{51}$ Our fitting software uses the Marquardt algorithm as outlined in P. R. Bevington, Data Reduction and Error Analysis for the Physical Sciences (McGraw-Hill, New York, 1969).

${ }^{52}$ D. Ross, D. Bonn, and J. Meunier, Nature (London) 400, 737 (1999).

${ }^{53}$ CRC Handbook of Chemistry and Physics, 75th edition, edited by D. R. Lide and H. P. R. Frederikse (CRC Press, Boca Raton, FL, 1994).

${ }^{54}$ P. Müller-Buschbaum, M. Tolan, and W. Press, Z. Phys. B: 95, 331 (1994).

${ }^{55}$ J. Bowers, E. Manzanares-Papayanopoulos, I. A. McLure, and R. Cubitt, J. Phys.: Condens. Matter 10, 8173 (1998).

${ }^{56}$ M. M. Neilson, J. Bowers, E. Manzanares-Papayanopoulos, J. R. Howse, M. C. Vergara-Gutierrez, P. J. Clements, A. N. Burgess, and I. A. McLure, Phys. Chem. Chem. Phys. 1, 4635 (1999).

${ }^{57}$ M. K. Kumaran and M. L. McGlashan, Aust. J. Chem. 33, 1909 (1980).

${ }^{58}$ Rotating anode data shows that both PF and MC alone wet our substrate at $30^{\circ} \mathrm{C}$.

${ }^{59}$ A. O. Parry and R. Evans, Phys. Rev. Lett. 64, 439 (1990); A. O. Parry and R. Evans, Physica A 181, 250 (1992).

${ }^{60}$ See, for example, A. M. Ferrenberg, D. P. Landau, and K. Binder, Phys. Rev. E 58, 3353 (1998), and references therein.

${ }^{61}$ A. Plech, U. Klemradt, M. Huber, and J. Peisl, Europhys. Lett. 49, 583 (2000). 